\title{
SOME SHARP RESTRICTION THEOREMS FOR HOMOGENEOUS MANIFOLDS
}

\author{
Laura De Carli and Alex Iosevich
}

Introduction: Let $M$ denote a submanifold of $\mathbb{R}^{n+l}$ of codimension $l$. Let $\mathcal{R}$ denote a restriction operator

$$
\mathcal{R} f(\eta)=\int e^{-i\langle x, \eta\rangle} f(x) d x, \quad \eta \in M, \quad f \in \mathcal{S}\left(\mathbb{R}^{n+l}\right) .
$$

We wish to find an optimal range of exponents $p$ such that

$$
\|\mathcal{R} f\|_{L^{2}(M, d \sigma)} \leq C_{p}\|f\|_{L^{p}\left(\mathbb{R}^{n+l}\right)},
$$

where $d \sigma$ is a compactly supported measure on $M$.

When $M$ is a codimension one surface in $\mathbb{R}^{n+1}$ with non-vanishing Gaussian curvature, the estimate (0.2) is well understood. A celebrated result due to Stein and Tomas says that if $M$ has non-vanishing Gaussian curvature, then the estimate $(0.2)$ holds with $p=\frac{2(n+2)}{n+4}$. In higher dimensions the situation is more complicated. When $M$ is a codimension two surface in $\mathbb{R}^{n+2}$ satisfying a non-degeneracy assumption, the estimate $(0.2)$ holds with $p=\frac{2(n+4)}{n+8}$. (See $[\mathrm{MC}]$ and Theorem A in the Section 2 below). However, a sharp necessary and sufficient condition is not currently available.

It should also be noted that even in codimension one, the more general $\left(L^{p}, L^{q}\right)$ estimates for the restriction operator are not fully understood, except in the dimension two. (See [St] for a detailed discussion). We shall not address this issue here.

The purpose of this paper is to establish the estimate $(0.2)$ in the case when

$$
M=\left\{\left(x, x_{k+1}, \ldots, x_{k+l}\right): x_{k+1}=\phi_{1}(x), \ldots, x_{k+l}=\phi_{l}(x)\right\}
$$

where $\phi_{j} \in C^{\infty}\left(\mathbb{R}^{n} \backslash 0\right)$ is homogeneous of degree $m_{j} \geq 1$.

Let $\mathcal{F}[d \sigma]$ denote the Fourier transform of $d \sigma$. By a theorem of Greenleaf (see $[\mathrm{Gr}]$ ), the inequality $(0.2)$ holds for $p=\frac{2(l+\gamma)}{2 l+\gamma}$ if

$$
|\mathcal{F}[d \sigma](R \zeta)| \leq C(1+R)^{-\gamma}, \quad \zeta \in S^{n+1}
$$


We shall see below that isotropic Fourier transform estimates do not yield the sharp restriction theorem in codimension two or higher (see e.g [MC]). It should be noted that even in codimension one, it is not known whether the exponent given by Greenleaf's theorem is sharp.

The best possible isotropic rate of decay of $\mathcal{F}[d \sigma]$ for the homogeneous manifold $M$ defined above is $-\frac{n}{\max _{j}\left\{m_{j}\right\}}$. An application of Greenleaf's theorem yields the estimate (0.2) with

$$
p_{0}=\frac{2(n+l m)}{n+2 l m}
$$

where $m=\max _{j}\left\{m_{j}\right\}$.

However, the following homogeneity argument due to Knapp suggests that the optimal exponent for the estimate $(0.2)$ is

$$
p_{0}=\frac{2\left(m_{1}+\ldots+m_{l}+n\right)}{n+2\left(m_{1}+\ldots+m_{l}\right)} .
$$

Indeed, let $\mathcal{R}$ denote the restriction operator defined above. Let $\hat{f}_{\delta}=h$, where $h$ is the characteristic function of a rectangle in $\mathbb{R}^{n+l}$ with sides of lengths $(1,1, \cdots 1, C, \ldots, C), C$ large.

Then

$$
\left\|f_{\delta}\right\|_{p} \approx \delta^{(1-1 / p)\left(n+m_{1}+\ldots+m_{l}\right)} \quad \text { and } \quad\left\|\mathcal{R} f_{\delta}\right\|_{2} \approx \delta^{n / 2}
$$

Hence $(0.2)$ can only hold if $p \leq \frac{2\left(n+m_{1}+\ldots+m_{l}\right)}{n+2\left(m_{1}+\ldots+m_{l}\right)}$.

We will establish the estimate (0.2) for a homogeneous manifold $M$, with the exponent $p_{0}$ given by Knapp's homogeneity argument, under a variety of conditions on the level sets of the graphing functions $\phi_{1}, \phi_{2}, \ldots, \phi_{l}$.

Our main results are the following. The first result gives us a good description of $L^{2}$ restriction theorems for two-dimensional submanifolds in codimension 2 given as graphs of homogeneous polynomials. (Please see Definition 2.1 and 2.2 below for the precise description of finite type. Please see Definition 2.8 below for the description of the order of vanishing along a line.)

Theorem I. (See Theorem 2.9 in Section 2). Let

$$
S=\left\{\left(x_{1}, x_{2}, x_{3}, x_{4}\right): x_{3}=\phi_{1}\left(x_{1}, x_{2}\right), x_{4}=\phi_{2}\left(x_{1}, x_{2}\right),\right\}
$$

where $\phi_{1}, \phi_{2} \in C^{\infty}\left(\mathbb{R}^{2}\right)$ are homogeneous polynomials of degree $m_{1}$ and $m_{2}$ respectively, $\left(m_{1} \geq m_{2} \geq 2\right)$. Suppose that there exists a non-zero constant $c$ such that $\phi_{1}(x)_{\left.\right|_{\left\{x: \phi_{2}(x)=1\right\}}=}=$ c. Let $Z_{0}=\left\{\left(x_{1}, x_{2}\right): \phi_{2}\left(x_{1}, x_{2}\right)=0\right\}, Z_{1}=\left\{\left(x_{1}, x_{2}\right): \nabla \phi_{2}\left(x_{1}, x_{2}\right)=(0,0)\right.$, and $Z_{2}=$ 
$\left\{\left(x_{1}, x_{2}\right): H \phi_{2}\left(x_{1}, x_{2}\right)=0\right\}$, where $H \phi_{2}$ denotes the determinant of the hessian matrix of $\phi_{2}$. Suppose that the curve $\left\{x: \phi_{2}(x)=1\right\}$ is of finite type $m$ at each point of $Z_{2} \cap\{x$ : $\left.\phi_{2}(x)=1\right\}$ and that $\phi_{2}$ vanishes of order $\leq M$ along the lines contained in $Z_{0} \cup Z_{1}$. Then (0.2) holds for every $p<p_{0}$, where $p_{0}$ is the sharp exponent

$$
\frac{m_{1}+m_{2}+2}{m_{1}+m_{2}+1}
$$

if $m_{1}+m_{2} \geq \max \left\{2 M\left(1+\frac{m_{1}}{m_{2}}\right), 2 m\right\}$. Furthermore (0.2) holds with

(1) $p<\max \left\{\frac{2\left(1+M\left(1+\frac{m_{1}}{m_{2}}\right)\right.}{1+2 M\left(1+\frac{m_{1}}{m_{2}}\right)}, \frac{2(m+1)}{2 m+1}\right\}$ if $\min \left\{2 M\left(1+\frac{m_{1}}{m_{2}}\right), 2 m\right\} \leq m_{1}+m_{2}<$ $\max \left\{2 M\left(1+\frac{m_{1}}{m_{2}}\right), 2 m\right\}$,

(2) $p<\min \left\{\frac{2\left(1+M\left(1+\frac{m_{1}}{m_{2}}\right)\right.}{1+2 M\left(1+\frac{m_{1}}{m_{2}}\right)}, \frac{2(m+1)}{2 m+1}\right\}$ if $4 \leq m_{1}+m_{2}<\min \left\{2 M\left(1+\frac{m_{1}}{m_{2}}\right), 2 m\right\}$.

The following result answers a question posed by Fulvio Ricci about the restriction theorems for manifolds given as graphs of quadratic monomials. The proof relies on the precise asymptotics of the Fourier transforms of certain distributions obtained by Shintani (see [TS], and Lemma 3.3 below). It has been brought to our attention that this result is implied by a theorem announced in the Bulletin of the AMS by Gerd Mockenhaupt (see [GM]). We enclose the proof for reader's convenience, and to motivate the related results proved in this paper. (See e.g. Theorem III below).

Theorem II. (See Theorem 3.1 below). Let $S=\left\{\left(x, x_{n+1}, \ldots, x_{n+l}\right) \in \mathbb{R}^{n+l}: x_{n+1}=\right.$ $\left.\phi_{1}(x), \ldots, x_{n+l}=\phi_{l}(x)\right\}$, where $l=\frac{n(n+1)}{2}$, and the $\phi_{j}$ denote the distinct monomials of degree 2. Then the estimate (0.2) holds with the sharp exponent $p_{0}=\frac{2(n+2)}{2 n+3}$.

The following result generalizes Theorem II to manifolds given as joint graphs of smooth functions of higher order of homogeneity. The proof relies on the non-isotropic decay estimates for the associated Fourier transform of the surface carried measure. The observation that the non-isotropic decay estimates are useful to obtain sharp restriction theorems in codimension $>1$ is not new. See for example the work of M.Christ $([\mathrm{MC}])$ and E.Prestini $([\mathrm{P}])$.

Theorem III. (See Theorem 3.2 below). Let $S$ denote a compact piece of the manifold $\left\{\left(x, x_{n+1}, \ldots, x_{n+l}\right) \in \mathbb{R}^{n+l}: x_{n+1}=\phi_{1}(x), \ldots, x_{n+l}=\phi_{l}(x)\right\}$, where $\phi_{j} \in C^{\infty}\left(\mathbb{R}^{n} / 0\right)$ is homogeneous of degree $m \geq 2 n$. Suppose that no linear combination of the $\phi_{j}$ 's vanishes on a subset of postive measure of $S^{n-1}$. Let $\Phi(x)=\left(\phi_{1}(x), \ldots, \phi_{l}(x)\right)$. Suppose that $\Phi(\omega) \neq$ $(0, \ldots, 0), \omega \in S^{n-1}$. Then the estimate $(0.2)$ holds with the sharp exponent

$$
p_{0}=\frac{2(n+l m)}{n+2 l m}
$$


Many of our results are based on the non-isotropic decay estimates for the associated Fourier transform of the surface carried measure. A sample result is the following.

Theorem IV. (See Theorem $1.1 B$ below) Let $S=\left\{\left(x, x_{n+1}, \ldots, x_{n+l}\right) \in \mathbb{R}^{n+l}: x_{n+1}=\right.$ $\left.\phi_{1}(x), \ldots, x_{n+l}=\phi_{l}(x)\right\}$, where each $\phi_{j} \in C^{\infty}\left(\mathbb{R}^{n} / 0\right)$ is homogeneous of degree $m \geq 2 n$. Let $\Phi(x)=\left(\phi_{1}(x), \ldots, \phi_{l}(x)\right)$. Let $\Phi_{\lambda}(x)=\langle\Phi(x), \lambda\rangle$. Let

$$
F(\xi, \lambda)=\int e^{\left.i\langle x, \xi\rangle+\lambda_{1} \phi_{1}(x)+\ldots+\lambda_{l} \phi_{l}(x)\right)} \psi(x) d x
$$

where $\psi$ is a smooth cutoff function. Then

$$
|F(\xi, \lambda)| \leq C \int_{S^{n-1}}\left|\Phi_{\lambda}(\omega)\right|^{-\frac{n}{m}} d \omega .
$$

This paper is organized as follows. In Section 1 we will prove some estimates related to the decay of the Fourier transform of the surface carried measure. In particular, we will estimate the decay of the Fourier transform of the surface carried measure, in any codimension, in terms of the integrability of the multiplicative inverses of the graphing functions $\phi_{j}$ restricted to the unit sphere. Using this technique we shall also obtain an accurate non-isotropic estimate for the Fourier transform of the surface carried measure in the case when every graphing function has the same homogeneity.

In Section 2 we will apply the results of Section 1 along with the results of M. Christ $([\mathrm{MC}])$, E. Prestini $([\mathrm{P}])$, and a variety of scaling arguments to obtain a sharp estimate $(0.2)$ with the exponent $p_{0}$ given by $(0.5)$.

In Section 3 we will use the non-isotropic estimates from Section 1 to study restriction theorems in the case when every graphing function is homogeneous of the same degree $m \geq 2$.

\section{SECTION 1}

The DECAY of The Fourier TRANSForm of THE SURFACE CARRIED MEASURE

We will need the following definitions. (See e.g. [Gr], [MC]).

Nonvanishing Gaussian curvature: Let $\Sigma$ be a submanifold of $\mathbb{R}^{N+1}$ of codimension 1 equipped with a smooth compactly supported measure $d \mu$. Let $J: \Sigma \rightarrow S^{N}$ be the usual Gauss map taking each point on $\Sigma$ to the outward unit normal at that point. We say that $\Sigma$ has everywhere nonvanishing Gaussian curvature if the differential of the Gauss map $d J$ is always nonsingular.

Strong curvature condition: Let $S$ be a submanifold of $\mathbb{R}^{n+l}$ of codimension $l$ equipped with a smooth compactly supported measure $d \mu$. Suppose that $S$ is a joint graph of smooth 
functions $g_{1}, g_{2}, \ldots, g_{l}$, where $g_{j}: \mathbb{R}^{n} \rightarrow \mathbb{R}$. Let $\mathcal{N}_{x_{0}}(S)$ denote the $l$-dimensional space of normals to $S$ at a point $x_{0}$. We say that $S$ satisfies the strong curvature condition (SCC) if for all $x_{0} \in S$ in some neighborhood of the support of $d \mu$,

$$
\operatorname{det} D^{2}\left(\nu_{1} g_{1}(x)+\nu_{2} g_{2}(x)+\ldots+\nu_{l} g_{l}(x)\right) \neq 0, \quad \forall \nu \in \mathcal{N}_{x_{0}},
$$

where $D^{2}$ denotes the Hessian matrix.

N-curvature condition: Let $S \in \mathbb{R}^{n+l}$ be defined as above. We say that $S$ satisfies the $N$-curvature condition if the rank of the Hessian matrix in (1.1) is greater than or equal to $N$ everywhere.

Our main results are the following.

Theorem 1.1A. Let $S=\left\{\left(x, x_{n+1}, \ldots, x_{n+l}\right) \in \mathbb{R}^{n+l}: x_{n+1}=\phi_{1}(x), \ldots, x_{n+l}=\phi_{n+l}(x)\right\}$, where $\phi_{j} \in C^{\infty}\left(\mathbb{R}^{n} \backslash 0\right)$ is homogeneous of degree $m_{j} \geq 2$. Suppose that no $\phi_{i}$ is a constant multiple of any $\phi_{j}$ for $i \neq j$. Suppose that $\left(\phi_{j}\right)^{-1} \in L^{\rho_{j}}\left(S^{n-1}\right), 0<\rho_{j} \leq \frac{n}{m_{j}}$. Let $\mu$ denote the number of distinct $m_{j}$ 's. Suppose that $\frac{n}{m_{j}} \leq \frac{1}{\mu+1}$ for each $j$. Let $\lambda=\left(\lambda_{1}, \ldots, \lambda_{l}\right)$, and define

$$
F(\xi, \lambda)=\int e^{i\left(\langle x, \xi\rangle+\lambda_{1} \phi_{1}(x)+\ldots+\lambda_{l} \phi_{l}(x)\right)} \psi(x) d x,
$$

where $\psi$ is a smooth cutoff function.

Then

$$
|F(\xi, \lambda)| \leq C(1+|\xi|+|\lambda|)^{-\rho}
$$

where $\rho=\min _{j}\left\{\rho_{j}\right\}$.

Theorem 1.1B. Let $S=\left\{\left(x, x_{n+1}, \ldots, x_{n+l}\right) \in \mathbb{R}^{n+l}: x_{n+1}=\phi_{1}(x), \ldots, x_{n+l}=\phi_{l}(x)\right\}$, where each $\phi_{j} \in C^{\infty}\left(\mathbb{R}^{n} / 0\right)$ is homogeneous of degree $m \geq 2$. Let $\Phi(x)=\left(\phi_{1}(x), \ldots, \phi_{l}(x)\right)$. Let $\Phi_{\lambda}(x)=\langle\Phi(x), \lambda\rangle$. Let $F(\xi, \lambda)$ be defined as in (1.2). Then

$$
|F(\xi, \lambda)| \leq C \int_{S^{n-1}}\left|\Phi_{\lambda}(\omega)\right|^{-\frac{n}{m}} d \omega
$$

Lemma 1.2. Let $S \in \mathbb{R}^{n+l}$ and $F(\xi, \lambda)$ be defined as above. Let

$$
F_{0}(\xi, \lambda)=\int e^{i\left(\langle x, \xi\rangle+\lambda_{1} \phi_{1}(x)+\ldots+\lambda_{l} \phi_{l}(x)\right)} \psi_{0}(x) d x
$$

where $\psi_{0}$ is a smooth cutoff function supported away from the origin, and each $\phi_{j} \in$ $C^{\infty}\left(\mathbb{R}^{n} \backslash 0\right)$ is homogeneous of degree $m \geq 2$. Suppose that

$$
\left|F_{0}(\xi, \lambda)\right| \leq C(1+|\xi|+|\lambda|)^{-\gamma}, \quad \gamma>0 .
$$

Then

$$
|F(\xi, \lambda)| \leq C(1+|\xi|+|\lambda|)^{-\frac{n}{\max _{j}\left\{m_{j}\right\}}},
$$

if $\gamma \max _{j}\left\{m_{j}\right\} \geq n$. 
Lemma 1.3. Let $S \in \mathbb{R}^{n+l}$ be defined as above. Suppose that $\left.S\right|_{\left\{x_{n+j_{1}}=1, x_{n+j_{2}}=1, \ldots, x_{n+j_{s}}=1\right\}}$, $1 \leq s<l$, is an $(n-s)$-dimensional submanifold of codimension $l$, of the hyperplane

$$
\left\{\left(x, x_{n+1}, \ldots, x_{n+l}\right): x_{n+j_{1}}=1, \ldots, x_{n+j_{s}}=1\right\}
$$

satisfying the $N$-curvature condition. Let $F_{0}(\xi, \lambda)$ be defined as in (1.7). Then

$$
\left|F_{0}(\xi, \lambda)\right| \leq C(1+|\xi|+|\lambda|)^{-\frac{N}{2}}
$$

Lemma 1.4. Let $S \in \mathbb{R}^{n+l}$ be defined as above with $l=2$. Suppose that there exists a constant $c$, such that $\left.\phi_{1}\right|_{\left\{x: \phi_{2}(x)=1\right\}} \equiv c$. Suppose that $m_{1} \neq m_{2}$. Suppose that H $\phi_{1}$, the Hessian matrix of $\phi_{1}$, has rank $\geq 2$ away from the origin. Suppose that $\phi_{1}(\omega) \neq 0$, $\omega \in S^{n-1}$. Let $F_{0}(\xi, \lambda)$ be defined as in (1.7). Then

$$
\left|F_{0}(\xi, \lambda)\right| \leq C(1+|\xi|+|\lambda|)^{-\frac{1}{2}}
$$

Lemma 1.5. Let $S \in \mathbb{R}^{n+l}$ be defined as in lemma 1.4 , with $n=l=2$. Then the assumptions of Theorem $B$ (see Section 2) are satisfied at every $x \neq(0,0)$ iff $\phi_{2}(\omega) \neq 0, \omega \in S^{1}$, and the level set $\left\{x: \phi_{2}=1\right\}$ has non-vanishing Gaussian curvature.

Proof of Theorem 1.1A. We shall prove the theorem under the assumption that all the $m_{j}$ 's are distinct. The general statement follows by combining the terms with the same homogeneity. Recall that

$$
F(\xi, \lambda)=\int e^{i\left(\langle x, \xi\rangle+\lambda_{1} \phi_{1}(x)+\ldots+\lambda_{l} \phi_{l}(x)\right)} \psi(x) d x,
$$

where without loss of generality $\psi$ is radial, and each $\phi_{j} \in C^{\infty}\left(\mathbb{R}^{n}\right)$ is homogeneous of degree $m_{j} \geq 2$.

Let $\Omega=\left\{\omega \in S^{n-1}:\left|\phi_{1}(\omega)\right|>\frac{1}{\left|\lambda_{1}\right|}, \ldots,\left|\phi_{l}(\omega)\right|>\frac{1}{\left|\lambda_{l}\right|}\right\}$.

Let

$$
F(\xi, \lambda)=\int_{\frac{x}{|x|} \in S^{n-1} \backslash \Omega}+\int_{\frac{x}{|x|} \in \Omega}=F_{S^{n-1} \backslash \Omega}+F_{\Omega} .
$$

Taking absolute values inside the integral we see that

$$
\left|F_{S^{n-1} \backslash \Omega}(\xi, \lambda)\right| \leq C\left|S^{n-1} \backslash \Omega\right|,
$$

which by Chebyshev's inequality is bounded by

$$
C \max _{j}\left\{\left|\lambda_{j}\right|^{-\rho_{j}} \int_{S^{n-1}}\left|\phi_{j}(\omega)\right|^{-\rho_{j}} d \omega\right\} \leq C|\lambda|^{-\rho},
$$


for $|\lambda|$ large.

Let $\psi_{0} \in C_{0}^{\infty}(\mathbb{R})$ be supported in the interval $\left[\frac{1}{2}, 4\right]$, such that $\psi_{0} \equiv 1$ inside $[1,2]$, and

$$
\sum_{k=0}^{\infty} \psi_{0}\left(2^{k} s\right) \equiv 1
$$

Let

$$
F_{\Omega}^{k}(\xi, \lambda)=\iint e^{i\left(r\langle\omega, \xi\rangle+\lambda_{1} r^{m_{1}} \phi_{1}(\omega)+\ldots+\lambda_{l} r^{m_{l}} \phi_{l}(\omega)\right)} \psi_{0}\left(2^{k} r\right) r^{n-1} d r d \omega
$$

A change of variables sending $r \rightarrow 2^{-k} r$ shows that

$$
F_{\Omega}^{k}(\xi, \lambda)=2^{-n k} F_{\Omega}^{0}\left(2^{-k} \xi, 2^{-m_{1} k} \lambda_{1}, \ldots, 2^{-m_{l} k} \lambda_{l}\right)
$$

Let $\mathcal{A}=\{1,2, \ldots, l\}$.

We must estimate

$$
\sum_{k=0}^{\infty} 2^{-n k}\left|F_{\Omega}^{0}\left(2^{-n k} \xi, 2^{-m_{1} k} \lambda_{1}, \ldots, 2^{-m_{l} k} \lambda_{l}\right)\right|=\sum_{\mathcal{B} \subset \mathcal{A}} I_{\mathcal{B}}
$$

where

$$
\begin{gathered}
I_{\mathcal{B}}=\sum_{\left\{\lambda_{j} \phi_{j}(\omega) \leq 2^{m_{j} k}, j \in \mathcal{B}, \lambda_{j} \phi_{j}(\omega)>2^{m_{j} k}, j \notin \mathcal{B}\right\}} 2^{-n k}\left|F_{\Omega}^{0}\left(2^{-k} \xi, 2^{-m_{1} k} \lambda_{1}, \ldots, 2^{-m_{l} k} \lambda_{l}\right)\right|= \\
\sum_{S_{\{\mathcal{B}, \leq\}} \cap S_{\{\mathcal{B},>\}} 2^{-n k}\left|F_{\Omega}^{0}\left(2^{-k} \xi, 2^{-m_{1}} k \lambda_{1}, \ldots, 2^{-m_{l} k} \lambda_{l}\right)\right| .}
\end{gathered}
$$

To estimate each $I_{\mathcal{B}}$ we shall need the fact that the curve $\left(r, r^{m_{1}}, \ldots, r^{m_{l}}\right)$ has nonvanishing curvature and torsion away from the origin, so long as all the $m_{j}$ 's are distinct. An elementary van der Corput type estimate shows that the Fourier transform of the measure carried by this curve decays of order $-\frac{1}{l+1}$. We shall also use the fact that $F_{\Omega}^{0}$ is bounded. More precisely,

$$
\begin{gathered}
\left|I_{\mathcal{B}}\right| \leq \int_{\Omega}\left(C \sum_{S_{\{\mathcal{B}, \leq\}}} 2^{-n k}\right)+\left(C \sum_{S_{\{\mathcal{B},>\}}} 2^{-n k}\left(\sum_{j \in \mathcal{B}} 2^{-m_{j} k}\left|\phi_{j}(\omega) \lambda_{j}\right|\right)^{-\frac{1}{l+1}}\right) d \omega \leq \\
\max _{j \in \mathcal{A}}\left|\lambda_{j}\right|^{-\frac{n}{m_{j}}} \int_{\Omega}\left|\phi_{j}(\omega)\right|^{-\frac{n}{m_{j}}} d \omega,
\end{gathered}
$$


provided that $\frac{n}{m_{j}} \leq \frac{1}{l+1}$, for each $j \in \mathcal{A}$.

Moreover,

$$
\begin{gathered}
\left|\lambda_{j}\right|^{-\frac{n}{m_{j}}} \int_{\Omega}\left|\phi_{j}(\omega)\right|^{-\frac{n}{m_{j}}} d \omega=\left|\lambda_{j}\right|^{-\rho_{j}} \int_{\Omega}\left|\phi_{j}(\omega)\right|^{-\rho_{j}}\left|\phi_{j}(\omega) \lambda_{j}\right|^{\rho_{j}-\frac{n}{m_{j}}} d \omega \leq \\
\left|\lambda_{j}\right|^{-\rho_{j}} \int_{S^{n-1}}\left|\phi_{j}(\omega)\right|^{-\rho_{j}} d \omega,
\end{gathered}
$$

since $\rho_{j} \leq \frac{n}{m_{j}}$.

Hence, the expression (1.21) is bounded by

$$
\max _{j \in \mathcal{A}}\left|\lambda_{j}\right|^{-\rho_{j}} \int_{S^{n-1}}\left|\phi_{j}(\omega)\right|^{-\rho_{j}} d \omega \leq C(|\lambda|)^{-\rho},
$$

for $|\lambda|$ large.

This completes the proof if $|\xi| \leq C|\lambda|$. However, if this is not the case, the gradient of the phase function $\langle x, \xi\rangle+\lambda_{1} \phi_{1}(x)+\ldots+\lambda_{l} \phi_{l}(x)$ is bounded away from zero, and an integration by parts argument (see [St], p.364) shows that $|F(\xi, \lambda)| \leq C_{N} 1+|\xi|+|\lambda|^{-N}$, for any $N>0$.

Proof of Theorem 1.1B. Let $\lambda=t \nu=t\left(\nu_{1}, \ldots, \nu_{l}\right)$. Let $\Phi_{\nu}(x)=\langle\Phi(x), \nu\rangle$. We rewrite $F(\xi, \lambda)$ in the form

$$
\int e^{i\left(\langle x, \xi\rangle+t \Phi_{\nu}(x)\right)} \psi(x) d x
$$

where $\psi$ is a smooth cutoff function. Let $\Omega=\left\{\omega \in S^{n-1}:\left|\Phi_{\nu}(\omega)\right|>\frac{1}{t}\right\}$. Let

$$
F(\xi, \lambda)=\int_{\frac{x}{|x|} \in S^{n-1} \backslash \Omega}+\int_{\frac{x}{|x|} \in \Omega}=F_{S^{n-1} \backslash \Omega}+F_{\Omega} .
$$

Taking the absolute values inside the integral, we see that

$$
\left|F_{S^{n-1} \backslash \Omega}(\xi, \lambda)\right| \leq C\left|S^{n-1} \backslash \Omega\right|=C\left|\left\{\omega \in S^{n-1}:\left|\Phi_{\nu}^{-1}(\omega)\right|>t\right\}\right|,
$$

which by Chebyshev's inequality is bounded by

$$
t^{-\frac{n}{m}} \int\left|\Phi_{\nu}(\omega)\right|^{-\frac{n}{m}} d \omega
$$

Let $\psi_{0} \in C_{0}^{\infty}(\mathbb{R})$ be supported in the interval $\left[\frac{1}{2}, 4\right]$, such that $\psi_{0} \equiv 1$ inside $[1,2]$, and

$$
\sum_{k=0}^{\infty} \psi_{0}\left(2^{k} s\right) \equiv 1 .
$$


Let

$$
F_{\Omega}^{k}(\xi, \lambda)=\iint e^{i\left(r\langle\omega, \xi\rangle+t r^{m} \Phi_{\nu}(\omega)\right)} r^{n-1} \psi_{0}\left(2^{k} r\right) d \omega d r
$$

A change of variables sending $r \rightarrow 2^{-k} r$ shows that $F_{\Omega}^{k}(\xi, \lambda)=2^{-n k} F_{\Omega}^{0}\left(2^{-k} \xi, 2^{-m k} \lambda\right)$. We must estimate

$$
\sum_{k=0}^{\infty} 2^{-n k}\left|F_{\Omega}^{0}\left(2^{-k} \xi, 2^{-m k} \lambda\right)\right|=\sum_{\left\{t \Phi_{\nu}(\omega) \leq 2^{m k}\right\}}+\sum_{\left\{t \Phi_{\nu}(\omega)>2^{m k}\right\}}=I+I I .
$$

To estimate $I I$ we shall use the fact that away from zero the Fourier transform of the measure supported on the curve $\left(r, r^{m}\right)$ decays of order $-\frac{1}{2}$. To estimate $I$ we shall just use the fact that $F_{\Omega}^{0}$ is bounded. More precisely,

$$
|I| \leq C \int_{\Omega} \sum_{\left\{t \Phi_{\nu}(\omega) \leq 2^{m k}\right\}} 2^{-n k} d \omega \leq C t^{-\frac{n}{m}} \int\left|\Phi_{\nu}(\omega)\right|^{-\frac{n}{m}} d \omega
$$

and

$$
|I I| \leq C \int_{\Omega} \sum_{\left\{t \Phi_{\nu}(\omega)>2^{m k}\right\}} 2^{-n k}\left|2^{-m k} \Phi_{\nu}(\omega) t\right|^{-\frac{1}{2}} d \omega \leq C t^{-\frac{n}{m}} \int_{\Omega}\left|\Phi_{\nu}(\omega)\right|^{-\frac{n}{m}} d \omega
$$

as long as $m \geq 2 n$.

This completes the proof if $|\xi| \leq C|\lambda|$. However, if this is not the case, the gradient of the phase function $\langle x, \xi\rangle+\lambda_{1} \phi_{1}(x)+\ldots+\lambda_{l} \phi_{l}(x)$ is bounded away from the origin, and an integration by parts argument (see [St], p.364) shows that $|F(\xi, \lambda)| \leq C_{N}(1+|\xi|+|\lambda|)^{-N}$, for any $N>0$.

Proof of Lemma 1.2. Let

$$
F_{k}(\xi, \lambda)=\int e^{i\left(\langle x, \xi\rangle+\lambda_{1} \phi_{1}(x)+\ldots+\lambda_{l} \phi_{l}(x)\right)} \psi_{0}\left(2^{k} x\right) d x
$$

A change of variables sending $x \rightarrow 2^{-k} x$ shows that

$$
F_{k}(\xi, \lambda)=2^{-n k} F_{0}\left(2^{-k} \xi, 2^{-m_{1} k} \lambda_{1}, \ldots, 2^{-m_{l} k} \lambda_{l}\right) .
$$

Let $\mathcal{A}=\{1,2, \ldots, l\}$. We must estimate

$$
\sum_{k=0}^{\infty} 2^{-n k}\left|F_{0}\left(2^{-k} \xi, 2^{-m_{1} k} \lambda_{1}, \ldots, 2^{-m_{l} k} \lambda_{l}\right)\right|=\sum_{\mathcal{B} \subset \mathcal{A}} I_{\mathcal{B}}
$$


where

$$
\begin{gathered}
I_{\mathcal{B}}=\sum_{\left\{\left|\lambda_{j}\right| \leq 2^{m_{j} k}, j \in \mathcal{B},\left|\lambda_{j}\right|>2^{m_{j} k}, j \notin \mathcal{B}\right\}} 2^{-n k}\left|F_{0}\left(2^{-k} \xi, 2^{-m_{1} k} \lambda_{1}, \ldots, 2^{-m_{l} k} \lambda_{l}\right)\right|= \\
\sum_{S_{\{\mathcal{B}, \leq\}} \cap S_{\{\mathcal{B},>\}}} 2^{-n k}\left|F_{0}\left(2^{-k} \xi, 2^{-m_{1} k} \lambda_{1}, \ldots, 2^{-m_{l} k} \lambda_{l}\right)\right| .
\end{gathered}
$$

Using the assumed decay of $F_{0}$, and the fact that, in particular, $F_{0}$ is bounded, we get

$$
\begin{gathered}
\left|I_{\mathcal{B}}\right| \leq C_{1} \sum_{S_{\{\mathcal{B}, \leq\}}} 2^{-n k}+C_{2} \sum_{S_{\{\mathcal{B},>\}}} 2^{-n k}\left(\sum_{j \notin \mathcal{B}} 2^{-m_{j} k}\left|\lambda_{j}\right|\right)^{-\gamma} \leq \\
C \max _{j \in \mathcal{A}}\left|\lambda_{j}\right|^{-\frac{n}{m_{j}}} \leq C|\lambda|^{-\frac{n}{\max _{j}\left\{m_{j}\right\}}},
\end{gathered}
$$

as long as $\max _{j}\left\{m_{j}\right\} \leq n \gamma$.

This completes the proof if $|\xi| \leq C|\lambda|$. However, if this is not the case, the gradient of the phase function $\langle x, \xi\rangle+\lambda_{1} \phi_{1}(x)+\ldots+\lambda_{l} \phi_{l}(x)$ is bounded away from the origin. An integration by parts argument, (see [St], p.364), shows that $|F(\xi, \lambda)| \leq C_{N}(1+|\xi|+|\lambda|)^{-N}$, for any $N>0$.

Proof of lemma 1.3. Assume that $x_{n+j_{1}}=x_{n+1}, \cdots x_{n+j_{s}}=x_{n+s}$. In what follows we will denote $x \in \mathbb{R}^{n}$ by $\left(x^{\prime}, x^{\prime \prime}\right)$, with $x^{\prime} \in \mathbb{R}^{s}, x^{\prime \prime} \in \mathbb{R}^{n-s}$ and by $J_{x^{\prime}}(f)$ (resp. $J_{x^{\prime \prime}}(f)$ ) and $D_{x^{\prime}}^{2} g$ (resp. $D_{x^{\prime \prime}}^{2} g$ ) the Jacobian of a function $f\left(x^{\prime}, x^{\prime \prime}\right)$ and the Hessian matrix of a function $g\left(x^{\prime}, x^{\prime \prime}\right)$ computed with respect to the $x^{\prime}$ (resp. $\left.x^{\prime \prime}\right)$ variables. Let $\phi=\left(\phi_{1}, \ldots, \phi_{s}\right)$.

Take $P$ on the support of $d \sigma$ such that $\phi(P)=(1,1, \ldots, 1)$. Since $S_{\left.\right|_{\left\{x_{n+1}=1, \cdots x_{n+s}=1\right\}}}$ is by assumption a submanifold of codimension $l$ of the hyperplane $\left\{\left(x, x_{n+1}, \cdots, x_{n+l}\right): x_{n+1}=\right.$ $\left.1, \cdots x_{n+s}=1\right\}$, the Jacobian of the function $\phi, J(\phi)$, has rank $s$ at $P$.

There is no loss of generality if we assume that $J_{x^{\prime}} \phi(P)$ is the identity in the space of $s \times s$ matrices, and that $J_{x^{\prime \prime}} \phi(P)=0$

By the implicit function theorem there exists a smooth function $\psi\left(x^{\prime \prime}\right): \mathbb{R}^{n-s} \rightarrow \mathbb{R}^{s}$ such that $\phi\left(\psi\left(x^{\prime \prime}\right), x^{\prime \prime}\right) \equiv 1$ in a neighborhood of $P^{\prime \prime}$.

An application of the chain rule yields:

(1) $J \phi\left(\psi\left(x^{\prime \prime}\right), x^{\prime \prime}\right)=J_{x^{\prime}} \phi\left(\psi\left(x^{\prime \prime}\right), x^{\prime \prime}\right) \times J \psi\left(x^{\prime \prime}\right)+J_{x^{\prime \prime}} \phi\left(\psi\left(x^{\prime \prime}\right), x^{\prime \prime}\right) \equiv 0$, which implies that $J \psi\left(P^{\prime \prime}\right)=0$,

(2) $\frac{\partial}{\partial} x_{s+j} J \psi\left(P^{\prime \prime}\right)+\frac{\partial}{\partial} x_{s+j} J_{x^{\prime \prime}} \phi(P)=0$ for every $j \leq n+s$, and

$$
\begin{aligned}
& D^{2}\left(\phi_{s+j}\right)\left(\psi\left(x^{\prime \prime}\right), x^{\prime \prime}\right)_{\left.\right|_{x^{\prime \prime}}=P^{\prime \prime}}=\sum_{k=1}^{s} \frac{\partial}{\partial} x_{k} \phi_{s+j}\left(\psi\left(x^{\prime \prime}\right), x^{\prime \prime}\right)_{x_{x^{\prime \prime}=P^{\prime \prime}}} D^{2}\left(\psi_{k}\right)\left(P^{\prime \prime}\right) \\
& \quad+D_{x^{\prime \prime}}^{2} \phi_{s+j}\left(\psi\left(x^{\prime \prime}\right), x^{\prime \prime}\right) \text { for every } j \leq n-s .
\end{aligned}
$$


¿From (2) we have that, for every $k \leq s$, the Hessian matrix of $\psi_{k}$ at $P^{\prime \prime}, D^{2} \psi_{k}\left(P^{\prime \prime}\right)$, is $-D_{x^{\prime \prime}}^{2} \phi_{k}\left(P^{\prime \prime}\right)$, and from $(3)$ that $D_{x^{\prime \prime}}^{2} \phi_{s+j}(P)$ can be written as a linear combination of the Hessian matrices of the functions $\psi_{k}$ and of the Hessian matrix of $\phi_{s+j}\left(\psi\left(x^{\prime \prime}\right), x^{\prime \prime}\right)$ at $P^{\prime \prime}$.

Let $\Phi=\langle x, \xi\rangle+\lambda_{1} \phi_{1}+\cdots \lambda_{l} \phi_{l}$ denote the phase function of $F$ as in (1.2). By the above remark, $D_{x^{\prime \prime}}^{2} \Phi(P)$ can be written as a linear combination of the Hessian matrices of the functions $\psi_{k}\left(x^{\prime \prime}\right)$ and the function $\phi_{s+j}\left(\psi\left(x^{\prime \prime}\right), x^{\prime \prime}\right)$ at $P^{\prime \prime}$. Since $S_{\left.\right|_{\left\{x_{n+1}=1, \cdots x_{n+s}=1\right\}} \text { satisfies }}$ the $N$-curvature condition, the rank of every linear combination of the above matrices is $N$ for every $\lambda \neq 0$. This shows that the rank of the Hessian matrix of $\Phi$ is $\geq N$ and hence that (1.9) holds.

Proof of Lemma 1.4. A theorem of Littman $([\mathrm{L}])$ says that if a surface in codimension one has at least $k$ non-vanishing principal curvatures, then the Fourier transform of the surface carried measure decays of order $-\frac{k}{2}$. The proof of that theorem shows that $F_{0}$ has the required decay if the rank of the Hessian matrix of the phase function $\Phi(x)=\langle x, \xi\rangle+$ $\lambda_{1} \phi_{1}(x)+\lambda_{2} \phi_{2}(x)$ is $\geq 1$ for every $x$ on the support of $\chi$ and for every $\left(\lambda_{1}, \lambda_{2}\right) \neq(0,0)$.

We observe now that $\phi_{1}(x) \equiv c \phi_{2}^{\frac{m_{1}}{m_{2}}}(x)$. Indeed, if $\phi_{2}\left(x_{0}\right) \neq 0$, then $\phi_{2}\left(\frac{x_{0}}{\phi_{2}\left(x_{0}\right)^{\frac{1}{m_{2}}}}\right)=$ 1 , and since $\left.\phi_{1}\right|_{\left\{\phi_{2}(x)=1\right\}} \equiv c$, then $\phi_{1}\left(\frac{x_{0}}{\phi_{2}\left(x_{0}\right)^{\frac{1}{m_{2}}}}\right)=\frac{\phi_{1}\left(x_{0}\right)}{\phi_{2}\left(x_{0}\right)^{\frac{m_{1}}{m_{2}}}}=c$. Then $\phi_{1}\left(x_{0}\right)=$ $c \phi_{2}\left(x_{0}\right)^{\frac{m_{1}}{m_{2}}}$ for every $x_{0} \in \mathbb{R}^{n}$ such that $\phi_{2}\left(x_{0}\right) \neq 0$. But the argument that we have just used shows that $\phi_{2}\left(x_{0}\right) \neq 0$ iff $\phi_{1}\left(x_{0}\right) \neq 0$. Hence $\phi_{1}(x)=c \phi_{2}(x)^{\frac{m_{1}}{m_{2}}}$ for every $x \in \mathbb{R}^{n}$.

By the chain rule,

$$
D_{i, j} \phi_{1}(x)=c \frac{m_{1}}{m_{2}} D_{i, j} \phi_{2}(x) \phi_{2}(x)^{\frac{m_{1}}{m_{2}}-1}+c \frac{m_{1}}{m_{2}}\left(\frac{m_{1}}{m_{2}}-1\right) D_{i}\left(\phi_{2}\right) D_{j}\left(\phi_{2}\right) \phi_{2}(x)^{\frac{m_{1}}{m_{2}}-2},
$$

for $i, j, \leq n$. Then

$$
\begin{gathered}
D^{2}(\Phi)(x)=\left(\lambda_{2}+c \lambda_{1} \frac{m_{1}}{m_{2}} \phi_{2}(x)^{\frac{m_{1}}{m_{2}}-1}\right) D^{2}\left(\phi_{2}\right)(x)+ \\
c \lambda_{1} \frac{m_{1}}{m_{2}}\left(\frac{m_{1}}{m_{2}}-1\right) \phi_{2}(x)^{\frac{m_{1}}{m_{2}}-2}\left(D_{1}\left(\phi_{2}\right)(x) \nabla\left(\phi_{2}\right)(x) \cdots D_{n}\left(\phi_{2}\right)(x) \nabla\left(\phi_{2}\right)(x)\right) .
\end{gathered}
$$

Let $P$ be a point on the support of $\chi$. If we set $\lambda_{2}+c \lambda_{1} \frac{m_{1}}{m_{2}} \phi_{2}(P)^{\frac{m_{1}}{m_{2}}-1}=0$, we observe that $D^{2}(\Phi)(P)$ equals a matrix whose rank is 1 if and only if $\nabla\left(\phi_{2}\right)(P) \neq(0, \cdots, 0)$, $\phi_{2}(P)^{\frac{m_{1}}{m_{2}}-2} \neq 0$, and zero elsewhere. Since we assumed that $\phi_{2}$ doesn't vanish away from the origin, Euler's homogeneity relations guarantee that $\nabla\left(\phi_{2}\right)(P)$ doesn't vanish. Consequently, the rank of $D^{2}(\Phi)(P)$ is at most 1 .

To show that $D^{2}(\Phi)(P)$ cannot be zero, we observe that if this were the case we would have 
$(1.41)$

$$
-\frac{\left(\lambda_{2}+c \lambda_{1} \frac{m_{1}}{m_{2}} \phi_{2}(P)^{\frac{m_{1}}{m_{2}}-1}\right)}{c \lambda_{1} \frac{m_{1}}{m_{2}}\left(\frac{m_{1}}{m_{2}}-1\right) \phi_{2}(P)^{\frac{m_{1}}{m_{2}}-2}} D^{2} \phi_{2}(P)=\left(D_{1}\left(\phi_{2}\right)(P) \nabla\left(\phi_{2}\right)(P) \ldots D_{n}\left(\phi_{2}\right)(P) \nabla\left(\phi_{2}\right)(P)\right) \text {. }
$$

The coefficient which multiplies $D^{2} \phi_{2}(P)$ cannot be zero because the matrix on the righthand side has rank one. On the other hand, the matrix on the left-hand side has rank $\geq 2$ by assumption, hence the equality in (1.41) can never hold.

This concludes the proof of the theorem.

Proof of Lemma 1.5. After perhaps rotating and dilating the coordinates, we can work in a neighborhood of the point $(0,1)$.

Since there exists a constant $c$ such that $\left.\phi_{1}\right|_{\left\{x: \phi_{2}=1\right\}} \equiv c, \phi_{1} \equiv c \phi_{2}^{\frac{m_{2}}{m_{2}}}$. (See proof of Lemma 1.4).

By the chain rule $D_{i, j} \phi_{1}\left(x_{1}, x_{2}\right)=$

$$
\frac{m_{1}}{m_{2}} D_{i, j} \phi_{2}\left(x_{1}, x_{2}\right) \phi_{2}\left(x_{1}, x_{2}\right)^{\frac{m_{1}}{m_{2}}-1}+\frac{m_{1}}{m_{2}}\left(\frac{m_{1}}{m_{2}}-1\right) D_{i}\left(\phi_{2}\right) D_{j}\left(\phi_{2}\right) \phi_{2}\left(x_{1}, x_{2}\right)^{\frac{m_{1}}{m_{2}}-2},
$$

for $i, j, \leq 2$.

Observe that by Euler's homogeneity relations

(1) $D_{1}\left(\phi_{2}\right)(0,1)=m_{2} \phi_{2}(0,1)$,

(2) $D_{2}\left(\phi_{2}\right)(0,1)=\frac{1}{m_{2}-1} D_{12} \phi_{2}(0,1)$, and

(3) $D_{11}\left(\phi_{2}\right)(0,1)=m_{2}\left(m_{2}-1\right) \phi_{2}(0,1)$.

In order to show that the sufficient condition of Theorem $1.1 \mathrm{~B}$ is verified, we must show that the determinant of the matrix $J(x, y)$, whose rows are $\nabla\left(\frac{x_{1}^{2}}{2} D_{11} \phi_{1}(0,1)\right.$

$\left.+\frac{x_{2}^{2}}{2} D_{22} \phi_{1}(0,1)+x_{1} x_{2} D_{12} \phi_{1}(0,1)\right)$ and $\nabla\left(\frac{x_{1}^{2}}{2} D_{11} \phi_{2}(0,1)+\frac{x_{2}^{2}}{2} D_{22} \phi_{2}(0,1)+x_{1} x_{2} D_{12} \phi_{2}(0,1)\right)$, is not a square. A direct computations shows that the discriminant of the determinant of $J\left(x_{1}, x_{2}\right)$ is

$$
\frac{m_{1}^{2}\left(\frac{m_{1}}{m_{2}}-1\right)^{2}}{\left(m_{2}-1\right)^{2}}\left(\phi_{2}(0,1)\right)^{2\left(\frac{m_{1}}{m_{2}}-1\right)}\left[\operatorname{det}\left(H\left(\phi_{2}\right)\right)(0,1)\right]^{2}
$$

which doesn't vanish by the assumptions on $\phi_{2}$. Hence, $J^{-a}$ is integrable for $a<1$. This concludes the proof of the lemma. 


\section{SECTION 2}

\section{RESTRICTION THEOREMS-SCALING}

We will need the following results.

\section{Theorem A (M. Christ $([\mathrm{MC}]))$. Let}

$$
S=\left\{\left(x, x_{n+1}, x_{n+2}\right) \in \mathbb{R}^{n+2}: x_{n+1}=g_{1}(x), \quad x_{n+2}=g_{2}(x)\right\},
$$

where $g_{j} \in C^{\infty}\left(\mathbb{R}^{n}\right)$.

Suppose that (0.2) holds with $p_{0}=\frac{2(n+4)}{n+8}$. Then for every $k \leq \frac{n}{2}$ and every $\theta$, the expression

$$
\left(\frac{d}{d \theta}\right)^{k}\left(\operatorname{det} D^{2}\left(\cos (\theta) g_{1}(x)+\sin (\theta) g_{2}(x)\right)\right)
$$

does not vanish.

Theorem B (E. Prestini $([\mathrm{P}]))$. Let

$$
S=\left\{\left(x, x_{n+1}, \ldots, x_{2 n}\right) \in \mathbb{R}^{n+n}: x_{n+1}=g_{1}(x), \ldots, x_{2 n}=g_{n}(x)\right\},
$$

where $g_{j} \in C^{\infty}\left(\mathbb{R}^{n}\right)$. Let $G_{j}$ denote the quadratic part of the Taylor expansion of $g_{j}$. Suppose that the vectors $\left\{\nabla\left(\frac{\partial}{\partial x_{i}} G_{j}\right)(0)\right\}_{\substack{i \leq n \\ j \leq n}}$ span $\mathbb{R}^{n}$. Let $J(x)$ denote the determinant of the matrix $\left(\nabla_{x} G_{1}, \ldots, \nabla_{x} G_{n}\right)$. Suppose that $J^{-a} \in L^{1}\left(S^{n-1}\right)$, for any $a<1$. Then $(0.2)$ holds with $p_{0}=\frac{6}{5}$.

Note that when $n=2$, the assumptions of Theorem B are equivalent to the necessary condition (2.2) in Theorem A. In particular, the conditions of Theorem B are necessary and sufficient in that case.

Before stating our main results, we need to introduce the following definitions.

Definition 2.1. Let $\Gamma: I \rightarrow \mathbb{R}^{2}$, where $I$ is a compact interval in $\mathbb{R}$, and $\Gamma$ is smooth. We say that $\Gamma$ is finite type if $\left\langle\left(\Gamma(x)-\Gamma\left(x_{0}\right)\right), \mu\right\rangle$ does not vanish of infinite order for any $x_{0} \in I$, and any unit vector $\mu$.

We will also need a more precise definition to specify the order of vanishing at each point. Let $a_{0}$ denote a point in the compact interval $I$. We can always find a smooth function $\gamma$, such that in a small neighborhood of $a_{0}, \Gamma(s)=(s, \gamma(s))$, where $s \in I$.

Definition 2.2. Let $\Gamma$ be defined as before. Let $\Gamma(s)=(s, \gamma(s))$ in a small neighborhood of $a_{0}$. We say that $\Gamma$ is finite type $m$ at $a_{0}$ if $\gamma^{(k)}\left(a_{0}\right)=0$ for $1 \leq k<m$, and $\gamma^{(m)}\left(a_{0}\right) \neq 0$.

Our main results are the following. 
Lemma 2.3. Let $S=\left\{\left(x, x_{n+1}, \cdots, x_{n+l} \in \mathbb{R}^{n+l}: x_{n+1}=\phi_{1}(x), \cdots, x_{n+l}=\phi_{n}(x)\right\}\right.$, where each $\phi_{j} \in \mathcal{C}^{\infty}\left(\mathbb{R}^{n} /\{0\}\right)$, homogeneous of degree $m_{j} \geq 1$. Let $d \sigma$ denote a compactly supported smooth measure on $S$, and let $d \sigma_{0}=\chi(x) d \sigma$, where $\chi(x)$ is a smooth cutoff function supported away from the origin. Let $T f(x)=f * \widehat{d \sigma}$ and let $T_{0} f(x)=f * \widehat{d} \sigma_{0}$. Suppose that $T_{0}: L^{q_{0}}\left(\mathbb{R}^{n+l}\right) \rightarrow L^{q_{0}^{\prime}}\left(\mathbb{R}^{n+l}\right)$ is a bounded operator. Then $T: L^{p_{0}\left(m_{1}, \cdots m_{l}\right)}\left(\mathbb{R}^{n+l}\right) \rightarrow$ $L^{p_{0}^{\prime}\left(m_{1}, \cdots m_{l}\right)}\left(\mathbb{R}^{n+l}\right)$ is a bounded operator, where

$$
p_{0}\left(m_{1}, \cdots, m_{l}\right)=\frac{2\left(n+m_{1}+\cdots+m_{l}\right)}{n+2\left(m_{1}+\cdots+m_{l}\right)},
$$

as long as $p_{0}\left(m_{1}, \cdots, m_{l}\right) \leq q_{0}$.

Theorem 2.4. Let

$$
S=\left\{\left(x, x_{n+1}, \ldots, x_{n+l}\right): x_{n+1}=\phi_{1}(x), \ldots, x_{n+l}=\phi_{l}(x)\right\},
$$

where $\phi_{j} \in C^{\infty}\left(\mathbb{R}^{n} \backslash 0\right)$ is homogeneous of degree $m_{j} \geq 2$. Suppose that $S$ satisfies the assumptions of Theorem 1.1A. Then the estimate (0.2) holds with

$$
p_{0}=\frac{2(l+\rho)}{2 l+\rho} .
$$

Theorem 2.5. Let

$$
S=\left\{\left(x, x_{n+1}, \ldots, x_{n+l}\right): x_{n+1}=\phi_{1}(x), \ldots, x_{n+l}=\phi_{l}(x)\right\},
$$

where $\phi_{j} \in C^{\infty}\left(\mathbb{R}^{n} \backslash 0\right)$ is homogeneous of degree $m_{j} \geq 2$. Suppose that $S$ satisfies the assumptions of the Lemma 1.3. Then the estimate (0.2) holds with the sharp exponent

$$
p_{0}=\frac{2\left(n+m_{1}+\ldots+m_{l}\right)}{n+2\left(m_{1}+\ldots+m_{l}\right)},
$$

provided that $\left(m_{1}+\ldots+m_{l}\right) \geq \frac{2 l n}{N}$.

Theorem 2.6. Let

$$
S=\left\{\left(x, x_{n+1}, x_{n+2}\right): x_{n+1}=\phi_{1}(x), x_{n+2}=\phi_{2}(x)\right\}, n>2,
$$

where $\phi_{j} \in C^{\infty}\left(\mathbb{R}^{n} \backslash 0\right)$ is homogeneous of degree $m_{j} \geq 2$. Suppose that there exists a nonzero constant $c$, such that

$$
\left.\phi_{1}\right|_{\left\{x: \phi_{2}(x)=1\right\}} \equiv c .
$$

Suppose that $\phi_{1}(\omega) \neq 0, \omega \in S^{n-1}$. If the rank of the Hessian matrix of $\phi_{1}$ is $\geq 2$, then (0.2) holds with $p_{0}=\frac{2\left(m_{1}+m_{2}+n\right)}{n+2\left(m_{1}+m_{2}\right)}$, provided that $m_{1}+m_{2} \geq 4 n$.

In order to introduce the Theorem 2.9 below, we need the following result which was stated and proved by the second author in ([I2]). 
Lemma 2.7. Let $Z_{0}=\left\{\left(x_{1}, x_{2}\right): P\left(x_{1}, x_{2}\right)=0\right\}$. Let $Z_{1}=\left\{\left(x_{1}, x_{2}\right): \nabla P\left(x_{1}, x_{2}\right)=\right.$ $(0,0)\}$. Let $Z_{2}=\left\{\left(x_{1}, x_{2}\right): H P\left(x_{1}, x_{2}\right)=0\right\}$, where $H P\left(x_{1}, x_{2}\right)$ denotes the determinant of the Hessian matrix of $P$. Then for each $j=0,1,2, Z_{j}=\{(0,0)\} \cup \bigcup_{k=1}^{N_{j}} L_{k}$, where each $L_{k}$ is a line through the origin, and $N_{j}<\infty$. Moreover, $Z_{1}=Z_{0} \bigcap Z_{2}$.

Proof of Lemma 2.7. Let $P_{j}$ denote the partial derivative of $P$ with respect to $x_{j}$. Since $P$ is homogeneous of degree $m, P_{j}$ is homogeneous of degree $m-1$, and $\operatorname{HP}\left(x_{1}, x_{2}\right)$ is homogeneous of degree $2(m-2)$. By homogeneity, if $Z_{j}$ contains a point $\left(x_{1}, x_{2}\right)$, it also contains a line through the origin containing that point. Since $P$ is a polynomial, there can be at most a finite number of such lines. This proves the first assertion of the lemma.

By the Euler homogeneity relations,

$$
\begin{gathered}
m P\left(x_{1}, x_{2}\right)=x_{1} P_{1}\left(x_{1}, x_{2}\right)+x_{2} P_{2}\left(x_{1}, x_{2}\right), \\
(m-1) P_{1}\left(x_{1}, x_{2}\right)=x_{1} P_{11}\left(x_{1}, x_{2}\right)+x_{2} P_{12}\left(x_{1}, x_{2}\right),
\end{gathered}
$$

and

$$
(m-1) P_{2}\left(x_{1}, x_{2}\right)=x_{1} P_{21}\left(x_{1}, x_{2}\right)+x_{2} P_{22}\left(x_{1}, x_{2}\right),
$$

where the $\left\{P_{j k}\right\}$ denote the second partial derivatives. Hence, $Z_{0} \subset Z_{1}$. If we write the equations for $P_{1}$ and $P_{2}$ in matrix form we see that $(m-1) \triangle P\left(x_{1}, x_{2}\right)$ is obtained by applying the Hessian matrix of $P$ to the vector $\left(x_{1}, x_{2}\right)$. Hence, $Z_{2} \subset Z_{1}$. Putting these observations together we see that $Z_{0} \cap Z_{2} \subset Z_{1}$.

Suppose that both $P$ and $H P$ vanish along a line through the origin, which without loss of generality we take to be the $x_{1}$-axis. Then $m P\left(x_{1}, 0\right)=x_{1} P_{1}\left(x_{1}, 0\right)$. This implies that $P_{1}\left(x_{1}, 0\right)=0$. Also, $(m-1) P_{1}\left(x_{1}, 0\right)=x_{1} P_{11}\left(x_{1}, 0\right)$. This implies that $P_{11}\left(x_{1}, 0\right)=0$. $P_{2}\left(x_{1}, 0\right)=x_{1} P_{12}\left(x_{1}, 0\right)$. By assumption,

$$
H P\left(x_{1}, 0\right)=P_{11}\left(x_{1}, 0\right) P_{22}\left(x_{1}, 0\right)-P_{12}^{2}\left(x_{1}, 0\right)=0 .
$$

Since $P_{11}\left(x_{1}, 0\right)=0$, we must conclude that $P_{12}\left(x_{1}, 0\right)=0$, which implies that $P_{2}\left(x_{1}, 0\right)=0$. This proves that $\nabla P\left(x_{1}, 0\right)=(0,0)$ and hence that $Z_{1} \subset Z_{0} \cap Z_{2}$. This completes the proof of the lemma.

We shall need the following definition:

Definition 2.8 Let $f \in C^{\infty}\left(\mathbb{R}^{2}\right)$. We say that $f$ vanishes of order $M$ along the line $L=\left\{\left(x_{1}, x_{2}\right): x_{1}=s_{1} t, x_{2}=s_{2} t, t \in \mathbb{R}\right\}$ if $M$ is the largest positive integer so that $f\left(s_{1} t, s_{2} t\right)=t^{M} g(t)$, where $g \in C^{\infty}\left(\mathbb{R}^{2}\right)$ is allowed to vanish only at the origin. 
Theorem 2.9. Let $S=\left\{\left(x_{1}, x_{2}, x_{3}, x_{4}\right)\right.$ : $x_{3}=\phi_{1}\left(x_{1}, x_{2}\right), x_{4}=\phi_{2}\left(x_{1}, x_{2}\right)$, $\}$ where $\phi_{1}$, $\phi_{2} \in C^{\infty}\left(\mathbb{R}^{2}\right)$ are homogeneous polynomials of degree $m_{1}$ and $m_{2}$ respectively, $\left(m_{1} \geq m_{2} \geq\right.$ $2)$. Suppose that there exists a non-zero constant c such that $\phi_{1}(x)_{\left.\right|_{\left\{x: \phi_{2}(x)=1\right\}}}=c$. Let $Z_{0}$, $Z_{1}, Z_{2}$ be defined as in Lemma 2.7 with respect to $\phi_{2}$. Suppose that the curve $\left\{x: \phi_{2}(x)=1\right\}$ is of finite type $m$ at each point of $Z_{2} \cap\left\{x: \phi_{2}(x)=1\right\}$ and that $\phi_{2}$ vanishes of order $\leq M$ along the lines contained in $Z_{0} \cup Z_{1}$. Then (0.2) holds for every $p<p_{0}$, where $p_{0}$ is the sharp exponent

$$
\frac{m_{1}+m_{2}+2}{m_{1}+m_{2}+1}
$$

if $m_{1}+m_{2} \geq \max \left\{2 M\left(1+\frac{m_{1}}{m_{2}}\right), 2 m\right\}$. Furthermore (0.2) holds with

(1) $p<\max \left\{\frac{2\left(1+M\left(1+\frac{m_{1}}{m_{2}}\right)\right.}{1+2 M\left(1+\frac{m_{1}}{m_{2}}\right)}, \frac{2(m+1)}{2 m+1}\right\}$ if $\min \left\{2 M\left(1+\frac{m_{1}}{m_{2}}\right), 2 m\right\} \leq m_{1}+m_{2}<$ $\max \left\{2 M\left(1+\frac{m_{1}}{m_{2}}\right), 2 m\right\}$,

(2) $p<\min \left\{\frac{2\left(1+M\left(1+\frac{m_{1}}{m_{2}}\right)\right.}{1+2 M\left(1+\frac{m_{1}}{m_{2}}\right)}, \frac{2(m+1)}{2 m+1}\right\}$ if $4 \leq m_{1}+m_{2}<\min \left\{2 M\left(1+\frac{m_{1}}{m_{2}}\right), 2 m\right\}$.

Proof of Lemma 2.3. Let

$$
\widehat{d \sigma}(\xi, \lambda)=\int_{\mathbb{R}^{n}} e^{i\left(\langle x, \xi\rangle+\lambda_{1} \phi_{1}(x)+\cdots+\lambda_{l} \phi_{l}(x)\right)} \psi(x) d x
$$

where $\psi(x)$ is a cutoff function. Let $\rho$ be a cutoff function supported in the interval $(1,2)$ such that $\sum_{j=0}^{+\infty} \rho\left(2^{j} t\right)=1$ for every $t$, and let

$$
\widehat{d \sigma_{j}}(\xi, \lambda)=\int_{\mathbb{R}^{n}} e^{i\left(\langle x, \xi\rangle+\lambda_{1} \phi_{1}(x)+\cdots+\lambda_{l} \phi_{l}(x)\right)} \rho\left(2^{j} t\right) d x
$$

If we make the change of variables sending $x \rightarrow 2^{-j} x$ we can write:

$$
\widehat{d \sigma_{j}}(\xi, \lambda)=2^{-j n} \widehat{d \sigma_{0}}\left(2^{-j} \xi, 2^{-m_{1} j} \lambda_{1}, \cdots 2^{-m_{l} j} \lambda_{l}\right)
$$

Let $\tau_{j}$ denote the nonisotropic dilation

$$
\tau_{j} f\left(\xi, \lambda_{1}, \cdots, \lambda_{l}\right)=f\left(2^{j} \xi, 2^{m_{1} j} \lambda_{1}, \cdots, 2^{m_{l} j} \lambda_{l}\right)
$$

Then

$$
\widehat{d \sigma_{j}} * f=2^{-n j}\left(\tau_{-j} \widehat{d \sigma_{0}} * f\right)(\xi, \lambda)=2^{-n j}\left(\tau_{-j} \widehat{d \sigma_{0}} *\left(\tau_{-j} \tau_{j} f\right)\right)(\xi, \lambda) .
$$


A change of variables shows that $\tau_{-j} \widehat{d \sigma_{0}} *\left(\tau_{-j} \tau_{j} f\right)=2^{j\left(n+m_{1}+\cdots m_{l}\right)} \tau_{-j}\left(\widehat{d \sigma_{0}} * \tau_{j} f\right)$. It follows that

$$
\begin{gathered}
\left\|\widehat{d \sigma_{j}} * f\right\|_{q_{0}^{\prime}}=2^{-n j+j\left(n+m_{1}+\cdots m_{l}\right)}\left\|\tau_{-j}\left(\widehat{d \sigma_{0}} * \tau_{j} f\right)\right\|_{q_{0}^{\prime}}= \\
=2^{j\left(m_{1}+\cdots m_{l}\right)} 2^{\frac{j}{q_{0}^{\prime}}\left(n+m_{1}+\cdots m_{l}\right)}\left\|\widehat{d \sigma_{0}} *\left(\tau_{j} f\right)\right\|_{q_{0}^{\prime}} \leq \\
\leq C 2^{j\left(m_{1}+\cdots m_{l}\right)} 2^{\frac{j}{q_{0}^{\prime}}\left(n+m_{1}+\cdots m_{l}\right)}\left\|\tau_{j} f\right\|_{q_{0}}=C 2^{j\left(m_{1}+\cdots m_{l}\right)} 2^{j\left(\frac{1}{q_{0}^{\prime}}-\frac{1}{q_{0}}\right)\left(n+m_{1}+\cdots m_{l}\right)}\|f\|_{q_{0}} .
\end{gathered}
$$

The series $\sum_{j=1}^{\infty}\left\|\widehat{d \sigma_{j}} * f\right\|_{q_{0}^{\prime}}$ converges, provided that

$$
m_{1}+\cdots m_{l}+\left(\frac{1}{q_{0}^{\prime}}-\frac{1}{q_{0}}\right)\left(n+m_{1}+\cdots m_{l}\right)<0
$$

which yields

$$
q_{0}<\frac{2\left(n+m_{1}+\cdots m_{l}\right)}{n+2\left(m_{1}+\cdots m_{l}\right)}
$$

This concludes the proof of the lemma.

Proof of Theorem 2.4. The application of Greenleaf's theorem (see (0.3) above) yields the result.

Proof of Theorem 2.5. Let $F_{0}$ be defined as in (1.7). By Lemma 1.3

$$
\left|F_{0}(\xi, \lambda)\right| \leq C(1+|\xi|+|\lambda|)^{-\frac{N}{2}}
$$

By a theorem of Greenleaf, (see (0.3) above), the inequality $(0.2)$ holds with $q_{0}=\frac{2(2 l+N)}{4 l+N}$. An application of Lemma 2.3 completes the proof.

Proof of Theorem 2.6. Let $F_{0}$ be defined as in (1.7). By Lemma 1.4

$$
\left|F_{0}(\xi, \lambda)\right| \leq C(1+|\xi|+|\lambda|)^{-\frac{1}{2}}
$$

Applying Greenleaf's theorem as above we get $q_{0}=\frac{10}{9}$. An application of Lemma 2.3 completes the proof.

Proof of Theorem 2.9. Let $\Sigma$ be the level set $\left\{x: \phi_{2}(x)=1\right\}$, and let

$$
\widehat{d \sigma}\left(\xi, \lambda_{1}, \lambda_{2}\right)=\int_{\mathbb{R}^{2}} e^{i\left(\langle x \xi\rangle+\lambda_{1} \phi_{1}(x)+\lambda_{2} \phi_{2}(x)\right)} \chi(x) d x
$$


where $\chi$ is a smooth cutoff function. Let $Z_{0}, Z_{1}$ and $Z_{2}$ be defined as in Lemma 2.7 with respect to $\phi_{2}$. Recall that $Z_{0} \cup Z_{1} \cup Z_{2}$ is the union of a finite number of lines through the origin.

Let $\left\{\Gamma_{j}(x)\right\}_{j \leq N_{1}}$ and $\left\{T_{j}(x)\right\}_{j \leq N_{2}}$ be two finite families of cones in $\mathbb{R}^{2}$ with the following properties:

i) Each $\Gamma_{j}(x)$ contains exactly one line of $Z_{0} \cup Z_{1}$, and each $T_{j}(x)$ contains exactly one line of $Z_{2}$

ii) $\Gamma_{j} \cap \Gamma_{i}=\{0,0\}$ if $i \neq j, T_{j} \cap T_{i}=\{0,0\}$ if $i \neq j$, and $\Gamma_{j} \cap T_{i}=\{0,0\}$.

Let $\alpha_{j}$ be the characteristic function of $\Gamma_{j}$ and let $\beta_{j}$ be the characteristic function of $T_{j}$. Then

$$
\begin{gathered}
\widehat{d \sigma}\left(\xi, \lambda_{1}, \lambda_{2}\right)=\int_{\mathbb{R}^{2}} e^{i\left(\langle x \xi\rangle+\lambda_{1} \phi_{1}(x)+\lambda_{2} \phi_{2}(x)\right)} \chi(x)\left(\sum_{j=1}^{N_{1}} \alpha_{j}(x)\right) d x+ \\
\int_{\mathbb{R}^{2}} e^{i\left(\langle x \xi\rangle+\lambda_{1} \phi_{1}(x)+\lambda_{2} \phi_{2}(x)\right)} \chi(x)\left(\sum_{j=1}^{N_{2}} \beta_{j}(x)\right) d x+ \\
\int_{\mathbb{R}^{2}} e^{i\left(\langle x \xi\rangle+\lambda_{1} \phi_{1}(x)+\lambda_{2} \phi_{2}(x)\right)} \chi(x)\left(1-\sum_{j=1}^{N_{1}} \alpha_{j}(x)-\sum_{j=1}^{N_{2}} \beta_{j}(x)\right) d x=\widehat{d \sigma}_{1}+\widehat{d \sigma}_{2}+\widehat{d \sigma}_{3} .
\end{gathered}
$$

We first consider

$$
\widehat{d \sigma}_{3}\left(\xi, \lambda_{1}, \lambda_{2}\right)=\int_{\mathbb{R}^{2}} e^{i\left(\langle x \xi\rangle+\lambda_{1} \phi_{1}(x)+\lambda_{2} \phi_{2}(x)\right)} \tilde{\eta}(x) \chi(x) d x
$$

where we have set $\tilde{\eta}(x)=1-\sum_{j=1}^{N_{1}} \alpha_{j}(x)-\sum_{j=1}^{N_{2}} \beta_{j}(x)$. On the support of $\tilde{\eta}$ the curvature of $\Sigma=\left\{x: \phi_{2}(x)=1\right\}$ never vanishes and $\phi_{2}$ vanishes only at zero.

We recall that by the Stein-Tomas observation, (0.2) is equivalent to the inequality

$$
\|\widehat{d \sigma} * f\|_{L^{p^{\prime}\left(\mathbb{R}^{4}\right)}} \leq C_{j}\|f\|_{L^{p}\left(\mathbb{R}^{4}\right)}
$$

Let $\rho$ be a smooth cutoff function supported in the interval $(1,2)$, such that $\sum_{j=0}^{+\infty} \rho\left(2^{j} t\right) \equiv 1$. Let

$$
\widehat{d \sigma_{3, j}}\left(\xi, \lambda_{1}, \lambda_{2}\right)=\int_{\mathbb{R}^{2}} e^{i\left(\langle x \xi\rangle+\lambda_{1} \phi_{1}(x)+\lambda_{2} \phi_{2}(x)\right)} \rho\left(2^{j} x\right) \tilde{\eta}(x) d x
$$

The assumptions of Lemma 1.5 are satisfied on the support of $\rho\left(2^{j} x\right) \eta(x)$, hence the inequality (2.26) holds for the measure $d \sigma_{3, j}$ with $p=\frac{6}{5}$. Since the sharp exponent $p_{0}$ cannot exceed $\frac{6}{5}$, the estimate $(2.26)$ holds for $p \leq p_{0}$, provided that $m_{1}+m_{2} \geq 4$. 
If we make the change of variables sending $x \rightarrow 2^{-j} x$, and if we observe that $\tilde{\eta}$ is invariant with respect to dilations, we see that

$$
\begin{aligned}
& \widehat{d \sigma_{3, j}}(\xi, \lambda)=2^{-2 j} \int_{\mathbb{R}^{2}} e^{i\left(\left\langle 2^{-j} x \xi\right\rangle+\lambda_{1} 2^{-m_{1} j} \phi_{1}(x)+\lambda_{2} 2^{-m_{2} j} \phi_{2}(x)\right)} \rho(x) \tilde{\eta}(x) d x= \\
& 2^{-2 j} \widehat{d \sigma_{3,0}}\left(2^{-j} \xi, 2^{-m_{1} j} \lambda_{1}, 2^{-m_{2} j} \lambda_{2}\right)
\end{aligned}
$$

Without loss of generality we can replace $\tilde{\eta}$ by a function $\eta \in C^{\infty}\left(\mathbb{R}^{n} /\{0\}\right)$, homogeneous of degree zero, whose support coincides with the support of $\tilde{\eta}$.

Let $\tau_{j}$ denote the nonisotropic dilation

$$
\tau_{j} f\left(\xi, \lambda_{1}, \lambda_{2}\right)=f\left(2^{j} \xi, 2^{m_{1} j} \lambda_{1}, 2^{m_{2} j} \lambda_{2}\right)
$$

Then

$$
\widehat{d \sigma_{3, j}} * f=2^{-2 j}\left(\tau_{-j} \widehat{d \sigma_{3,0}} * f\right)\left(\xi, \lambda_{1}, \lambda_{2}\right)=2^{-2 j}\left(\tau_{-j} \widehat{d \sigma_{3,0}} *\left(\tau_{-j} \tau_{j} f\right)\right)\left(\xi, \lambda_{1}, \lambda_{2}\right) .
$$

A change of variables shows that $\tau_{-j} \widehat{d \sigma_{3,0}} *\left(\tau_{-j} \tau_{j} f\right)=2^{j\left(2+m_{1}+m_{2}\right)} \tau_{-j}\left(\widehat{d \sigma_{3,0}} * \tau_{j} f\right)$. It follows that

$$
\begin{gathered}
\left\|\widehat{d \sigma_{3, j}} * f\right\|_{p^{\prime}}=2^{-2 j+j\left(2+m_{1}+m_{2}\right)}\left\|\tau_{-j}\left(\widehat{d \sigma_{3,0}} * \tau_{j} f\right)\right\|_{p^{\prime}}= \\
=2^{j\left(m_{1}+m_{2}\right)} 2^{\frac{j}{p^{\prime}}\left(2+m_{1}+m_{2}\right)}\left\|\widehat{d \sigma_{3,0}} *\left(\tau_{j} f\right)\right\|_{p^{\prime}} \leq \\
\leq C 2^{j\left(m_{1}+m_{2}\right)} 2^{\frac{j}{p^{\prime}}\left(2+m_{1}+m_{2}\right)}\left\|\tau_{j} f\right\|_{p}=C 2^{j\left(m_{1}+m_{2}\right)} 2^{j\left(\frac{1}{p^{\prime}}-\frac{1}{p}\right)\left(2+m_{1}+m_{2}\right)}\|f\|_{p} .
\end{gathered}
$$

The series $\sum_{j=1}^{\infty}\left\|\widehat{d \sigma_{3, j}} * f\right\|_{p^{\prime}}$ converges, provided that

$$
m_{1}+m_{2}+\left(\frac{1}{p^{\prime}}-\frac{1}{p}\right)\left(n+m_{1}+m_{2}\right)<0
$$

which yields $p<p_{0}$. Hence the measure $d \sigma_{3}$ satisfies the inequality $(2.26)$ with $p<p_{0}$.

We consider now $\widehat{d \sigma}_{2}\left(\xi, \lambda_{1}, \lambda_{2}\right)=\int_{\mathbb{R}^{2}} e^{i\left(\langle x \xi\rangle+\lambda_{1} \phi_{1}(x)+\lambda_{2} \phi_{2}(x)\right)} \chi(x)\left(\sum_{j=1}^{N_{2}} \beta_{j}(x)\right) d x$.

Let

$$
\widehat{d \mu}\left(\xi, \lambda_{1}, \lambda_{2}\right)=\int_{\mathbb{R}^{2}} e^{i\left(\langle x \xi\rangle+\lambda_{1} \phi_{1}(x)+\lambda_{2} \phi_{2}(x)\right)} \chi(x) \beta_{j}(x) d x
$$


where $j \leq N_{2}$ is fixed.

In the proof of lemma 1.4 we observed that $\phi_{1}(x)_{\left.\right|_{\Sigma}}=c$ implies that $\phi_{1}(x)=c \phi_{2}(x)^{\frac{m_{1}}{m_{2}}}$ for every $x \in \mathbb{R}^{2}$. Observe that $\Sigma$ is star-shaped with respect to the origin, because if $x_{0} \in \Sigma$, then for every $t \leq 1, \phi_{1}\left(t x_{0}\right)=t^{m_{1}} \leq 1$. In the polar coordinates associated to $\Sigma$,

$$
\widehat{d \mu}\left(\xi, \lambda_{1}, \lambda_{2}\right)=\int_{\Sigma} \int_{0}^{+\infty} e^{i\left(r\langle\omega \xi\rangle+c \lambda_{1} r^{m_{1}}+\lambda_{2} r^{m_{2}}\right)} r \chi(r \omega) \beta_{j}(\omega) d r d \omega
$$

Without loss generality $\chi$ is radial. Consider the (unique) point of $Z_{2} \cap \Sigma \cap T_{j}$, which can be taken to be $(0,0)$. Suppose that $\Sigma \cap T_{j}$ is supported in a sufficiently small neighborhood of $(0,0)$. Since $\Sigma$ is finite type $m$, it can be written as the graph of a smooth function $\psi(t)=t^{m} g(t)$, where $g(0) \neq 0$, and

$$
\widehat{d \mu}\left(\xi, \lambda_{1}, \lambda_{2}\right)=\int_{\mathbb{R}} \int_{0}^{+\infty} e^{i\left(r t \xi_{1}+r t^{m} g(t) \xi_{2}+c \lambda_{1} r^{m_{1}}+\lambda_{2} r^{m_{2}}\right)} r \chi(r) \beta_{j}\left(t, t^{m} g(t)\right) d r d t
$$

Let $\rho \in C^{\infty}\left(\mathbb{R}^{2}\right)$ be supported in $\left(\frac{1}{4}, 4\right)$, such that $\rho \equiv 1$ in $(1,2)$, and $\sum_{j=1}^{+\infty} \rho\left(2^{j} t\right) \equiv 1$. Let

$$
\widehat{d \mu_{j}}\left(\xi, \lambda_{1}, \lambda_{2}\right)=\int_{\mathbb{R}} \int_{0}^{+\infty} e^{i\left(r t \xi_{1}+r t^{m} g(t) \xi_{2}+c \lambda_{1} r^{m_{1}}+\lambda_{2} r^{m_{2}}\right)} \rho\left(2^{j} t\right) \chi(r) r d r d t
$$

The integral with respect to $t$ is supported over a dyadic piece of $\Sigma$ where the Gaussian curvature does not vanish. By Lemma 1.5 and the Stein-Thomas observation, the estimate (2.26) holds for the measure $d \mu_{j}$, for $p \leq p_{0}$, with a constant $C_{j}$.

In order to estimate $C_{j}$ we make a change of variables in the expression for $\widehat{d \mu_{j}}$ setting $s=2^{j} t$. We have

$$
\widehat{d \mu_{j}}\left(\xi, \lambda_{1}, \lambda_{2}\right)=2^{-j} \int_{\mathbb{R}} \int_{0}^{+\infty} e^{i\left(r 2^{-j} s \xi_{1}+r 2^{-m j} s^{m} g\left(2^{-j} t\right) \xi_{2}+c \lambda_{1} r^{m_{1}}+\lambda_{2} r^{m_{2}}\right)} \chi(r) \rho(s) r d r d s .
$$

Let $\tau_{j}$ be the nonisotropic dilation $\tau_{j}(f)\left(x_{1}, x_{2}, x_{3}, x_{4}\right)=f\left(2^{-j} x_{1}, 2^{-m j} x_{2}, x_{3}, x_{4}\right)$ and let

$$
\widehat{d \widetilde{\mu}_{j}}=\int_{\mathbb{R}} \int_{0}^{+\infty} e^{i\left(r s \xi_{1}+r s^{m} g\left(2^{-j} t\right) \xi_{2}+c \lambda_{1} r^{m_{1}}+\lambda_{2} r^{m_{2}}\right)} \chi(r) \rho(s) r d r d s
$$

Then

$$
\widehat{d \mu_{j}} * f=2^{-j}\left(\tau_{j}\left(\widehat{d \widetilde{\mu}_{j}}\right) * f\right)=2^{-j}\left(\tau_{j}\left(\widehat{d \widetilde{\mu}_{j}}\right) * \tau_{j}\left(\tau_{-j} f\right)\right)
$$


A change of variables shows that $\tau_{j}\left({\widehat{d \widetilde{\mu}_{j}}}\right) * \tau_{j} \tau_{-j} f=2^{j(m+1)} \tau_{j}\left(\widehat{d \widetilde{\mu}}_{j} * \tau_{-j} f\right)$, and that $\left\|\tau_{j} \psi\right\|_{L^{q}\left(\mathbb{R}^{4}\right)}=2^{j \frac{(m+1)}{q}}\|\psi\|_{L^{q}\left(\mathbb{R}^{4}\right)}$ for every $\psi \in L^{q}\left(\mathbb{R}^{4}\right)$ and $q \geq 1$. Then we can write the following string of inequalities:

$$
\begin{gathered}
\left\|\widehat{d \mu_{j}} * f\right\|_{L^{p^{\prime}\left(\mathbb{R}^{4}\right)}}=2^{j m}\left\|\tau_{j}\left(\widehat{d \widetilde{\mu}_{j}} * \tau_{-j} f\right)\right\|_{L^{p^{\prime}\left(\mathbb{R}^{4}\right)}}= \\
2^{j m+j \frac{(m+1)}{p^{\prime}}}\left\|\widehat{d \tilde{\mu}_{j}} * \tau_{j} f\right\|_{L^{p^{\prime}\left(\mathbb{R}^{4}\right)}} \leq 2^{j m+j \frac{(m+1)}{p^{\prime}}} C_{j}\left\|\tau_{j} f\right\|_{L^{p}\left(\mathbb{R}^{4}\right)}= \\
2^{j m+j(m+1)\left(\frac{1}{p^{\prime}}-\frac{1}{p}\right)} C_{j}\|f\|_{L^{p}\left(\mathbb{R}^{4}\right)} .
\end{gathered}
$$

We must prove that the constants $C_{j}$ in the above expression are uniformly bounded. In fact the sum $\sum_{j=1}^{+\infty}\left\|\widehat{d \mu_{j}} * f\right\|_{L^{p^{\prime}\left(\mathbb{R}^{4}\right)}}$ converges if $m+(m+1)\left(\frac{1}{p^{\prime}}-\frac{1}{p}\right)<0$, hence if $p<\frac{2(m+1)}{2 m+1}$. Since $\frac{2(m+1)}{2 m+1} \geq p_{0}$ when $m_{1}+m_{2} \geq 2 m$, the estimate $(2.26)$ holds for the measure $d \mu$, and consequently for the measure $d \sigma_{2}$, for $p \leq p_{0}$, provided that $m_{1}+m_{2} \geq 2 m$, and with $p=\frac{2(m+1)}{2 m+1}$ if $m_{1}+m_{2}<2 m$.

¿From the proof of the theorem of Greenleaf it follows that the bounds for the constants $C_{j}$ depend on a finite number of derivatives of the phase function of $\widehat{d \tilde{\mu}_{j}}, \Phi_{j}(r, s)=$ $r s \xi_{1}+r s^{m} g\left(2^{-j} t\right) \xi_{2}+c \lambda_{1} r^{m_{1}}+\lambda_{2} r^{m_{2}}$. Since $\Phi_{j}$ is a smooth function, then, for $j$ large, $D^{\beta} \Phi_{j}(r, s) \approx D^{\beta}\left(r s \xi_{1}+r s^{m} g(0) \xi_{2}+c \lambda_{1} r^{m_{1}}+\lambda_{2} r^{m_{2}}\right)$. This shows that the constant $C_{j}$ 's are uniformly bounded.

We now consider $\widehat{d \sigma}_{1}\left(\xi, \lambda_{1}, \lambda_{2}\right)=\int_{\mathbb{R}^{2}} e^{i\left(\langle x \xi\rangle+\lambda_{1} \phi_{1}(x)+\lambda_{2} \phi_{2}(x)\right)} \chi(x)\left(\sum_{j=1}^{N_{1}} \alpha_{j}(x)\right) d x$.

Fix $j \leq N_{1}$. After perhaps a rotation of coordinates we may assume that $\phi_{\left.2\right|_{\Gamma_{j}}}$ vanishes along the $x_{2}$ axis. Then $\phi_{2}\left(x_{1}, x_{2}\right)$ can be written as $x_{1}^{M} g\left(x_{1}, x_{2}\right)$, where $g$ does not vanish on the $x_{2}$ axis, (except perhaps at the origin), if $\Gamma_{j} \cap S^{1}$ is small enough.

Let

$$
\widehat{d \mu}\left(\xi, \lambda_{1}, \lambda_{2}\right)=\int_{\mathbb{R}^{2}} e^{i\left(\langle x \xi\rangle+c \lambda_{1} x_{1}^{\gamma M} g(x)^{\gamma}+\lambda_{2} x_{1}^{M} g(x)\right)} \chi(x) \alpha_{j}(x) d x
$$

where we have set $\gamma=\frac{m_{1}}{m_{2}}$. Let $\rho \in C^{\infty}\left(\mathbb{R}^{2}\right)$ be a cutoff function supported in $\left(\frac{1}{4}, 4\right)$, such that $\rho \equiv 1$ in $(1,2)$ and $\sum_{j=1}^{+\infty} \rho(t) \equiv 1$. Let

$$
\widehat{d \mu_{j}}(\xi, \lambda)=\int_{\mathbb{R}^{2}} e^{i\left(\langle x \xi\rangle+c \lambda_{1} x_{1}^{\gamma M} g^{\gamma}(x)+\lambda_{2} x_{1}^{M} g(x)\right)} \rho\left(2^{j} \frac{x_{1}}{x_{2}}\right) \chi(x) d x .
$$

The above integral is defined over a cone of $\mathbb{R}^{2}$ where the curvature of $\Sigma$ never vanishes, and $\phi_{2}$ vanishes only at the origin. 
Let $\rho_{0} \in C^{\infty}\left(\mathbb{R}^{2}\right)$ be supported in $\left(\frac{1}{4}, 4\right)$, such that $\rho \equiv 1$ in $(1,2)$, and $\sum_{k=0}^{+\infty} \rho_{0}\left(2^{k} t\right) \equiv 1$. Let

$$
\widehat{d \mu_{j, k}}(\xi, \lambda)=\int_{\mathbb{R}^{2}} e^{i\left(\langle x \xi\rangle+c \lambda_{1} x_{1}^{\gamma M} g^{\gamma}(x)+\lambda_{2} x_{1}^{M} g(x)\right)} \rho\left(2^{j} \frac{x_{1}}{x_{2}}\right) \rho_{0}\left(2^{k} x\right) d x
$$

The assumptions of Lemma 1.5 are satisfied on the support of $\rho_{0}\left(2^{k} x\right) \rho\left(2^{j} \frac{x_{1}}{x_{2}}\right)$, and hence the estimate (2.26) holds for the measure $d \mu_{j, k}$ with $p \leq p_{0}$.

If we make the change of variables sending $x \rightarrow 2^{-k} x$,

$$
\begin{gathered}
\widehat{d \mu_{j, k}}(\xi, \lambda)=2^{-2 k} \int_{\mathbb{R}^{2}} e^{i\left(\left\langle 2^{-k} x \xi\right\rangle+\lambda_{1} 2^{-m_{1} k} \phi_{1}(x)+\lambda_{2} 2^{-m_{2} k} \phi_{2}(x)\right)} \rho\left(2^{j} \frac{x_{1}}{x_{2}}\right) \rho_{0}(x) d x= \\
2^{-2 k} \widehat{d \mu_{j, 0}}\left(2^{-k} \xi, 2^{-m_{1} k} \lambda_{1}, 2^{-m_{2} k} \lambda_{2}\right) .
\end{gathered}
$$

Let $\tau_{k}$ denote the nonisotropic dilation

$$
\tau_{k} f\left(\xi, \lambda_{1}, \lambda_{2}\right)=f\left(2^{k} \xi, 2^{m_{1} k} \lambda_{1}, 2^{m_{2} k} \lambda_{2}\right)
$$

Then

$$
\widehat{d \mu_{j, k}} * f=2^{-2 k}\left(\tau_{-k} \widehat{d \mu_{0, k}} * f\right)\left(\xi, \lambda_{1}, \lambda_{2}\right)=2^{-2 k}\left(\tau_{-k} \widehat{d \mu_{0, k}} *\left(\tau_{-k} \tau_{k} f\right)\right)\left(\xi, \lambda_{1}, \lambda_{2}\right) .
$$

A change of variables shows that $\tau_{-k} \widehat{d \mu_{j, k}} *\left(\tau_{-k} \tau_{k} f\right)=2^{k\left(2+m_{1}+m_{2}\right)} \tau_{-k}\left(\widehat{d \mu_{j, k}} * \tau_{k} f\right)$. It follows that

$$
\begin{gathered}
\left\|\widehat{d \mu_{j, k}} * f\right\|_{p^{\prime}}=2^{-2 k+k\left(2+m_{1}+m_{2}\right)}\left\|\tau_{-k}\left(\widehat{d \mu_{j, k}} * \tau_{k} f\right)\right\|_{p^{\prime}}= \\
=2^{k\left(m_{1}+m_{2}\right)} 2^{\frac{k}{p^{\prime}}\left(2+m_{1}+m_{2}\right)}\left\|\widehat{d \mu_{j, k}} *\left(\tau_{k} f\right)\right\|_{p^{\prime}} \leq \\
\leq C 2^{k\left(m_{1}+m_{2}\right)} 2^{\frac{k}{p^{\prime}}\left(2+m_{1}+m_{2}\right)}\left\|\tau_{k} f\right\|_{p}=C 2^{k\left(m_{1}+m_{2}\right)} 2^{k\left(\frac{1}{p^{\prime}}-\frac{1}{p}\right)\left(2+m_{1}+m_{2}\right)}\|f\|_{p} .
\end{gathered}
$$

The series $\sum_{k=1}^{\infty}\left\|\widehat{d \mu_{j, k}} * f\right\|_{p^{\prime}}$ converges, provided that

$$
m_{1}+m_{2}+\left(\frac{1}{p^{\prime}}-\frac{1}{p}\right)\left(n+m_{1}+m_{2}\right)<0
$$

which yields $p<p_{0}$. The above argument shows that we can assume that the measure $d \mu_{j}$ is supported away from zero. Hence, by Lemma 1.5 and the above observation, the inequality 
(2.26) holds for the measure $d \mu_{j}$, for $p \leq p_{0}$, with a constant $C_{j}$. In order to estimate $C_{j}$ we perform the change of variables in (2.42) sending $x_{1} \rightarrow 2^{-j} x_{1}, x_{2} \rightarrow x_{2}$. We obtain

$$
\widehat{d \mu_{j}}\left(\xi_{1}, \xi_{2}, \lambda_{1}, \lambda_{2}\right)=2^{-j} \widehat{d \widetilde{\mu}_{j}}\left(2^{-j} \xi_{1}, \xi_{2}, 2^{-M \gamma j} \lambda_{1}, 2^{-M j} \lambda_{2}\right)
$$

where we have set

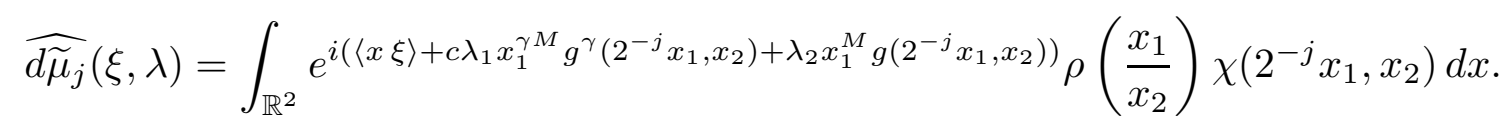

Let $\tau_{j}$ be the nonisotropic dilation $\tau_{j} f\left(x_{1}, x_{2}, x_{3}, x_{4}\right)=f\left(2^{-j} x_{1}, x_{2}, 2^{-M \gamma j} x_{3}, 2^{-M j} x_{4}\right)$. Then

$$
\widehat{d \mu_{j}} * f=2^{-j}\left(\tau_{j}\left(\widehat{d \widetilde{\mu}_{j}}\right) * f\right)=2^{-j}\left(\tau_{j}\left(\widehat{d \widetilde{\mu}_{j}}\right) * \tau_{j}\left(\tau_{-j} f\right)\right) .
$$

A change of variables shows that

$$
\tau_{j}\left(\widehat{d \widetilde{\mu}_{j}}\right) * \tau_{j}\left(\tau_{-j} f\right)=2^{j(1+M(1+\gamma))} \tau_{j}\left(\widehat{d \widetilde{\mu}_{j}} * \tau_{-j} f\right),
$$

and that $\left\|\tau_{j} g\right\|_{L^{q}\left(\mathbb{R}^{4}\right)}=2^{j \frac{(1+M(1+\gamma))}{q}}\|g\|_{L^{q}\left(\mathbb{R}^{4}\right)}$ for every $q \geq 1$. Then we can write the following string of inequalities:

$$
\begin{aligned}
& \left\|\widehat{d \mu}_{j} * f\right\|_{L^{p^{\prime}\left(\mathbb{R}^{4}\right)}}=2^{j(M(1+\gamma))}\left\|\tau_{j}\left(\widehat{d \widetilde{\mu}_{j}} * \tau_{-j} f\right)\right\|_{L^{p^{\prime}\left(\mathbb{R}^{4}\right)}}=
\end{aligned}
$$

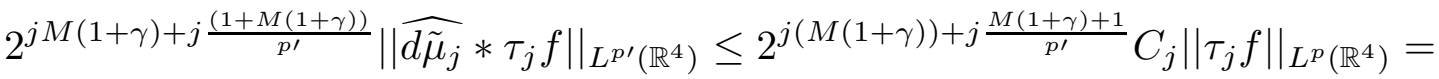

$$
\begin{aligned}
& 2^{j M(1+\gamma)+j(M(1+\gamma)+1)\left(\frac{1}{p^{\prime}}-\frac{1}{p}\right)} C_{j}\|f\|_{L^{p}\left(\mathbb{R}^{4}\right)} .
\end{aligned}
$$

If we show that the constants $C_{j}$ in the above expression are uniformly bounded then we are done. In fact the sum $\sum_{j=1}^{+\infty}\left\|\widehat{d \mu_{j}} * f\right\|_{L^{p^{\prime}\left(\mathbb{R}^{4}\right)}}$ converges if

$M(1+\gamma)+(M(1+\gamma)+1)\left(\frac{1}{p^{\prime}}-\frac{1}{p}\right)<0$ hence if

$$
p<\frac{2(1+M(\gamma+1))}{1+2 M(\gamma+1)} .
$$

Since $\frac{2(1+M(\gamma+1))}{1+2 M(\gamma+1)} \geq p_{0}$ when $m_{1}+m_{2} \geq 2 M(1+\gamma)$, the estimate (2.26) holds for the measure $d \mu$, and consequently for the measure $d \sigma_{1}$, with $p<p_{0}$, provided that $m_{1}+m_{2} \geq 2 M(1+\gamma)$, and with $p<\frac{2(1+M(\gamma+1)}{1+2 M(\gamma+1)}$ if $4<m_{1}+m_{2}<2 M(1+\gamma)$.

By a theorem of Greenleaf the bounds for the constants $C_{j}$ in (2.53) depend only on a finite number of derivatives of the phase function of $\widehat{d \tilde{\mu}_{j}}, \Phi_{j}(x)=\left(c \lambda_{1} x_{1}^{\gamma M} g^{\gamma}\left(2^{-j} x_{1}, x_{2}\right)+\right.$ $\left.\lambda_{2} x_{1}^{M} g\left(2^{-j} x_{1}, x_{2}\right)\right)$ Since the above function is smooth, then, for $j$ large, $D^{\beta} \Phi_{j}(x) \approx$ $D^{\beta}\left(c \lambda_{1} x_{1}^{\gamma M} g^{\gamma}\left(0, x_{2}\right)+\lambda_{2} x_{1}^{M} g\left(0, x_{2}\right)\right)$. This shows that the constants $C_{j}$ are uniformly bounded, thus concluding the proof of the theorem. 


\section{SeCtion 3}

\section{Restriction theOREMS- Non-ISOTROPIC ESTIMATES}

Theorem 3.1. Let $S=\left\{\left(x, x_{n+1}, \ldots, x_{n+l}\right) \in \mathbb{R}^{n+l}: x_{n+1}=\phi_{1}(x), \ldots, x_{n+l}=\phi_{l}(x)\right\}$, where $l=\frac{n(n+1)}{2}$, and the $\phi_{j}$ denote the distinct monomials of degree 2 . Then the estimate (0.2) holds with the sharp exponent $p_{0}=\frac{2(n+2)}{2 n+3}$.

Theorem 3.2. Let $S$ denote a compact piece of the manifold $\left\{\left(x, x_{n+1}, \ldots, x_{n+l}\right) \in \mathbb{R}^{n+l}\right.$ : $\left.x_{n+1}=\phi_{1}(x), \ldots, x_{n+l}=\phi_{l}(x)\right\}$, where $\phi_{j} \in C^{\infty}\left(\mathbb{R}^{n} / 0\right)$ is homogeneous of degree $m \geq 2 n$. Suppose that no linear combination of the $\phi_{j}$ 's vanishes on a subset of postive measure of $S^{n-1}$. Let $\Phi(x)=\left(\phi_{1}(x), \ldots, \phi_{l}(x)\right)$. Suppose that $\Phi(\omega) \neq(0, \ldots, 0), \omega \in S^{n-1}$. Then the estimate (0.2) holds with the sharp exponent $p_{0}$ given by (0.4).

Remark 1. The restriction $m \geq 2 n$ in Theorem 3.2 is not necessary. In fact, using the techniques in ([IS]), one can prove Theorem 3.2 under the weaker restriction $m \geq n$.

Remark 2. Theorem 3.2 implies the natural generalization of Theorem 3.1 to the case where $l=C_{m}^{m+n-1},\left(C_{b}^{a}=\frac{a !}{b !(a-b) !}\right)$, and the $\phi_{j}$ are the disitinct monomials of degree $m$.

Proof of Theorem 3.1. Let $\lambda=\left(\lambda_{1}, \cdots, \lambda_{l}\right)$, and let

$$
\widehat{d \sigma}(\xi, \lambda)=\int_{\mathbb{R}^{n}} e^{i\left(\langle x \xi\rangle+\lambda_{1} \phi_{1}+\cdots \lambda_{l} \phi_{l}(x)\right)} d x .
$$

Let $A_{\lambda}$ be the matrix associated to the quadratic form $\lambda_{1} \phi_{1}(x)+\cdots \lambda_{l} \phi_{l}(x)$, and

$$
\widehat{d \sigma}(\xi, \lambda)=\int_{\mathbb{R}^{n}} e^{i\left(\langle x \xi\rangle+\left\langle x, A_{\lambda} x\right\rangle\right)} d x
$$

Thus, $\widehat{d \sigma}(\xi, \lambda)$ is the Fourier transform of $e^{i\left\langle x, A_{\lambda} x\right\rangle}$, and an easy generalization of the wellknown formula for the Fourier transform of the Gaussian functions, (see e.g.[WR] pg. 186), yields

$$
\widehat{d \sigma}(\xi, \lambda)=\frac{(2 \pi)^{\frac{n}{2}}}{\left|\operatorname{det} A_{\lambda}\right|^{1 / 2}} e^{-\frac{i}{2}\left\langle\xi, A_{\lambda}^{-1} \xi\right\rangle+\frac{\pi i}{4} \operatorname{sign}\left(A_{\lambda}\right)}
$$

Let

$$
K_{z}(\xi, \lambda)=\psi(z) \operatorname{det}\left(A_{\lambda}\right)^{z} \widehat{d \sigma}(\xi, \lambda)=\psi(z)(2 \pi)^{\frac{n}{2}}\left|\operatorname{det} A_{\lambda}\right|^{z-1 / 2} e^{-\frac{i}{2}\left\langle\xi, A_{\lambda}^{-1} \xi\right\rangle+\frac{\pi i}{4} \operatorname{sign}\left(A_{\lambda}\right)}
$$

with

$$
\psi(z)=(2 \pi)^{n z+\frac{n(n+1)}{2}} \Pi_{j=0}^{n-1} \Gamma^{-1}\left(z+1+\frac{j}{2}\right) e^{-i \pi \frac{n}{2}\left(z+\frac{n+1}{2}\right)},
$$


where $\Gamma$ is the standard Gamma function. Let $T_{z}(f)(\xi, \lambda)=\left(f * K_{z}\right)(\xi, \lambda)$.

We will prove that $T_{z}$ is an continuous family of operators when $\operatorname{Re}(z) \in\left[-\frac{n+1}{2}, \frac{1}{2}\right]$, is analytic when $\operatorname{Re}(z) \in\left(-\frac{n+1}{2} \frac{1}{2}\right)$, and that

i) $\left\|T_{z}(f)\right\|_{L^{\infty}\left(\mathbb{R}^{n}\right)} \leq C_{1}(z)\|f\|_{L^{1}\left(\mathbb{R}^{n}\right)}$, when $\operatorname{Re}(z)=\frac{1}{2}$,

ii) $\left\|T_{z}(f)\right\|_{L^{2}\left(\mathbb{R}^{n}\right)} \leq C_{2}(z)\|f\|_{L^{2}\left(\mathbb{R}^{n}\right)}$, when $\operatorname{Re}(z)=-\frac{n+1}{2}$,

iii) $C_{1}(z)$ and $C_{2}(z)$ have at most exponential growth with respect to $\operatorname{Im}(z)$.

Stein's analytic interpolation theorem (see e.g. [So]) will then imply that $T_{z}: L^{p_{0}}\left(\mathbb{R}^{n+l}\right) \rightarrow L^{p_{0}^{\prime}}\left(\mathbb{R}^{n+l}\right)$ is a bounded operator when $z=0$. Since $T_{z}(f)=f * \widehat{d \sigma}$, the Stein-Thomas observation (see the proof of Theorem 2.9) implies the conclusion of our theorem.

To prove $(i)$ we observe that when $z=\frac{1}{2}+i y$, with $y \in \mathbb{R}$, then $K_{z}(\xi, \lambda)=\widehat{d \sigma}(\xi, \lambda) \operatorname{det}\left(A_{\lambda}\right)^{\frac{1}{2}+i y} \psi\left(\frac{1}{2}+i y\right)$ is bounded by $C_{1}(y)=\pi^{\frac{n}{2}}\left|\psi\left(\frac{1}{2}+i y\right)\right|$. By the Hausdorff -Young inequality we have that $\left\|f * K_{1+i y}\right\|_{\infty} \leq C_{1}(y)\|f\|_{1}$. (i) is then satisfied, and one can check, using Stirling's formula, that $C_{1}(y)$ has at most exponential growth.

To prove $(i i)$ it is enough to show that $\left|\widehat{K}_{z}\right|$ is a bounded function when $\operatorname{Re}(z)=-\frac{n+1}{2}$. To compute the the Fourier transform of $K_{z}(\xi, \lambda)$ with respect to $\xi, \mathcal{F}_{\xi}\left(K_{z}\right)(x, \lambda)$, we use again the formula (3.3) obtaining

$$
\mathcal{F}_{\xi}\left(K_{z}\right)(x, \lambda)=\psi(z)\left|\operatorname{det} A_{\lambda}\right|^{z} e^{i\left\langle x, A_{\lambda} x\right\rangle} .
$$

Hence

$$
\widehat{K_{z}}\left(x, x^{\prime}\right)=\psi(z) \int_{\mathbb{R}^{l}} e^{i\left(\left\langle x^{\prime}, \lambda\right\rangle+\left\langle x, A_{\lambda} x\right\rangle\right)} \operatorname{det}\left(A_{\lambda}\right)^{z} d \lambda,
$$

where we have set $x^{\prime}=\left(x_{n+1}, \cdots x_{n+l}\right)$. We recall that the above identities hold in distribution sense.

Since the phase of the above integral is a linear function of $\lambda$, we reduce to computing the Fourier transform of $\operatorname{det}\left(A_{\lambda}\right)^{z}$. We need the following lemma (see [TS], pg. 48).

Lemma 3.3. Let $V_{\mathbb{R}}$ be the space of the real and symmetric matrices and let $V_{i} \subset V_{\mathbb{R}}$ be the subset of the matrices with $i$ positive and $n-i$ negative eigenvalues. Let $G_{i}(z)$ be the distribution

$$
G_{i}(z)(f)=\int_{V_{i}} f(Y)|\operatorname{det}(Y)|^{z} d Y
$$

where $d Y$ is the standard Euclidean measure on $V_{\mathbb{R}}$. Then the distribution $G_{i}(z)$, viewed as a function of $z$, has analytic continuation to a meromorphic function in the whole complex plane satisfying

$$
\widehat{G_{i}(z)}=\psi^{-1}(z) \sum_{j=0}^{n} c_{i, j}(z) G_{j}\left(-z-\frac{n+1}{2}\right),
$$


where $\psi$ is as in (3.4) and the $C_{i, j}(z)$ are bounded coefficients.

¿From the above formula we deduce that, modulo bounded constants,

$$
\mathcal{F}\left(\operatorname{det}\left(A_{\lambda}\right)^{z}\right)(\eta)=\psi^{-1}(z) \operatorname{det}\left(A_{\eta}\right)^{-z-\frac{n+1}{2}},
$$

where the above formula holds in distribution sense. Since $\operatorname{Re}(z)=\frac{n+1}{2}$, the above is a bounded function of $\eta$. This shows that $\widehat{K_{z}}$ is a bounded function of $\left(x, x^{\prime}\right)$, and completes the proof of the theorem.

Proof of Theorem 3.2. Let

$$
\widehat{d \sigma}(\xi, \lambda)=\int_{\mathbb{R}^{n}} e^{i\left(\langle x \xi\rangle+\lambda_{1} \phi_{1}(x)+\lambda_{l} \phi_{l}(x)\right)} \chi(x) d x
$$

where $\chi$ is a smooth cutoff function. By Theorem $1.1 B$ we can write

$$
\widehat{d \sigma}(\xi, \lambda)=\widetilde{B}(\xi, \lambda)
$$

where $|\widetilde{B}(\xi, \lambda)| \leq \int_{S^{n-1}} \frac{d \omega}{\left|\sum_{j=1}^{l} \phi_{j}(\omega) \lambda_{j}\right|^{\frac{n}{m}}}$.

Let

$$
\begin{gathered}
B(\lambda)=\int_{S^{n-1}} \frac{d \omega}{\left|\sum_{j=1}^{l} \phi_{j}(\omega) \lambda_{j}\right|^{\frac{n}{m}}} \\
K_{z}(\xi, \lambda)=\widehat{d \sigma}(\xi, \lambda) B^{z}(\lambda) \psi(z)
\end{gathered}
$$

with

$$
\psi(z)=\Gamma^{-1}\left(-\frac{z n}{2 m}+\frac{1}{2}\right) \Gamma^{-1}\left(-\frac{n z}{m}+l\right),
$$

where $\Gamma$ is the standard Gamma function. Let $T_{z}(f)(\xi, \lambda)=\left(f * K_{z}\right)(\xi, \lambda)$.

We will prove that $T_{z}$ is a continous family of operators when $\operatorname{Re}(z) \in\left[-1, \frac{l m}{n}\right]$, is analytic when $\operatorname{Re}(z) \in\left(-1, \frac{l m}{n}\right)$, and that

i) $\left\|T_{z}(f)\right\|_{L^{\infty}\left(\mathbb{R}^{n}\right)} \leq C_{1}(z)\|f\|_{L^{1}\left(\mathbb{R}^{n}\right)}$, when $\operatorname{Re}(z)=-1$,

ii) $\left\|T_{z}(f)\right\|_{L^{2}\left(\mathbb{R}^{n}\right)} \leq C_{2}(z)\|f\|_{L^{2}\left(\mathbb{R}^{n}\right)}$, when $\operatorname{Re}(z)=\frac{m l}{n}$,

iii) $C_{1}(z)$ and $C_{2}(z)$ have at most exponential growth with respect to $\operatorname{Im}(z)$.

Stein's analytic interpolation theorem (see e.g. [So]) will then imply that $T_{z}: L^{p_{0}}\left(\mathbb{R}^{n+l}\right) \rightarrow L^{p_{0}^{\prime}}\left(\mathbb{R}^{n+l}\right)$ is a bounded operator when $z=0$. Since $T_{z}(f)=f * \widehat{d \sigma}$, Stein-Thomas observation (see the proof of Theorem 2.9) implies the conclusion of our theorem. 
To prove the estimate $(i)$ we observe that when $z=-1+i y$, with $y \in \mathbb{R}$, then $K_{z}(\xi, \lambda)=\widehat{d \sigma}(\xi, \lambda) B^{i y-1}(\lambda) \psi(-1+i y)$ is bounded by $C_{1}(y)=C\left|\psi_{1}(-1+i y)\right|$. By the Haussdorf -Young inequality we have that $\left\|f * K_{1+i y}\right\|_{\infty} \leq C_{1}(y)\|f\|_{1}$. The estimate $(i)$ is then satisfied, and one can check using Stirling's formula that $C_{1}(y)$ has at most exponential growth.

To prove the estimate $(i i)$ it is enough to show that $\widehat{K}_{z}$ is a bounded function when $\operatorname{Re}(z)=\frac{m l}{n}$. Since $d \sigma$ is a finite measure it is enough to prove that $\psi(z) \widehat{B^{z}}(\lambda)$ is bounded. Let $X(\omega)$ be the vector defined by the equation $\sum_{j=1}^{l} \lambda_{j} \phi_{j}(\omega)=\langle\lambda, X(\omega)\rangle$. Then

$$
\widehat{B_{z}}(y)=\psi(z) \int_{\mathbb{R}^{l}}\left(\int_{S^{n-1}} \frac{d \omega}{|\langle\lambda, X(\omega)\rangle|^{\frac{n}{m}}}\right)^{z} e^{i\langle\lambda, y\rangle} d \lambda
$$

Since $\widehat{B_{z}}(y)$ is homogeneous of degree zero with respect to $y$, we can assume $|y|=1$.

In polar coordinates with respect to $\lambda$, with $\lambda=r \eta$, we have

$$
\widehat{B_{z}}(y)=\psi(z) \int_{0}^{+\infty} r^{-\frac{n}{m} z+l-1} \int_{S^{l-1}}\left(\int_{S^{n-1}} \frac{e^{\frac{i}{z} r\langle\eta, y\rangle} d \omega}{|\langle\eta, X(\omega)\rangle|^{\frac{n}{m}}}\right)^{z} d \eta d r .
$$

Let $\chi(r) \in \mathcal{C}^{\infty}(\mathbb{R})$ be such that $\chi(r) \equiv 1$, when $r \in(0,1)$, and $\chi(r) \equiv 0$ when $r \in(2,+\infty)$. Let

$$
\widehat{B_{z, M}}(y)=\psi(z) \int_{0}^{+\infty} r^{-\frac{n}{m} z+l-1} \chi\left(\frac{r}{M}\right) \int_{S^{l-1}}\left(\int_{S^{n-1}} \frac{e^{\frac{i}{z} r\langle\eta, y\rangle} d \omega}{|\langle\eta, X(\omega)\rangle|^{\frac{n}{m}}}\right)^{z} d \eta d r
$$

We will prove that $\widehat{B_{z, M}}$ is bounded by a constant $C$ independent of $M$ and that $\widehat{B_{z, M}} \rightarrow \widehat{B_{z}}$ in distribution sense as $M \rightarrow \infty$. ¿From the above it follows that $\widehat{B_{z}}$ is bounded. Indeed, since the balls are sequentially compact in the weak $*$ topology in $L^{\infty}\left(\mathbb{R}^{l}\right)$, there exists a sequence $\left\{\widehat{B_{z, M_{j}}}\right\}_{j \in \mathbb{N}} \subset\left\{\widehat{B_{z, M}}\right\}$ which converges to a bounded function in the weak $*$ topology of $L^{\infty}$, and hence converges also in distribution sense. Consequently, $\widehat{B_{z}}=$ $\lim _{j \rightarrow+\infty} \widehat{B_{z, M_{j}}}$, which is a bounded function.

Recalling that by assumption the vector $X(\omega)$ is never zero on $S^{n-1}$, we can construct an orthogonal matrix $A_{\omega}$ with the property that $A_{\omega} \frac{X(\omega)}{|X(\omega)|}=(1,0, \cdots, 0)$. The first row of $A_{\omega}$ is $\frac{X(\omega)}{|X(\omega)|}$ and the other rows are a set of $l-1$ vectors which, togeter with $\frac{X(\omega)}{|X(\omega)|}$, determine an orthonormal basis of $\mathbb{R}^{l}$ for every $\omega \in S^{n-1}$.

We make the change of variables $\eta \rightarrow A_{\omega}^{t} \eta$ in the expression for $\widehat{B_{M, z}}$. Since $A_{\omega}$ is orthogonal, the change of variables maps $S^{l-1}$ into itself and the determinant of the Jacobian matrix of the transformation is 1 . We obtain

$$
\widehat{B_{z, M}}(y)=\psi(z) \int_{0}^{+\infty} r^{-\frac{n}{m} z+l-1} \chi\left(\frac{r}{M}\right) \int_{S^{l-1}}\left(\int_{S^{n-1}} \frac{|X(\omega)|^{\frac{n}{m}}}{\left|\left\langle\eta, A_{\omega} X(\omega)\right\rangle\right|^{\frac{n}{m}}} e^{\frac{i}{z} r\left\langle\eta, A_{\omega} y\right\rangle} d \omega\right)^{z} d \eta d r
$$




$$
=\psi(z) \int_{0}^{+\infty} r^{-\frac{n}{m} z+l-1} \chi\left(\frac{r}{M}\right) \int_{S^{l-1}} \frac{1}{\left|\eta_{1}\right|^{\frac{n z}{m}}}\left(\int_{S^{n-1}}|X(\omega)|^{\frac{n}{m}} e^{\frac{i}{z} r\left\langle\eta, A_{\omega} y\right\rangle} d \omega\right)^{z} d \eta d r
$$

The integral with respect to $\omega$ is a continous function of $r, \eta, y$, say $F(r, \eta, y)$. In particular $F(0, \eta, y)=\int_{S^{n-1}}|X(\omega)|^{\frac{n}{m}} d \omega<\infty$.

Since $\operatorname{Re}(z) \geq l$ by assumption, we can check that we can compute at least $l-1$ derivatives of $F(r, \eta, y)^{z}$ with respect to $r$ and $\eta$. Then

$$
\begin{gathered}
\widehat{B_{z, M}}(y)=\psi(z) \int_{0}^{+\infty} r^{-\frac{n}{m} z+l-1} \chi\left(\frac{r}{M}\right) \int_{S^{l-1}} \frac{1}{\left|\eta_{1}\right|^{\frac{n z}{m}}} F^{z}(r, \eta, y) d r d \eta= \\
\psi(z) \int_{0}^{+\infty} r^{-\frac{n}{m} z+l-1} \chi\left(\frac{r}{M}\right) \int_{-1}^{1} \int_{\left\{\left|\eta^{\prime}\right|=\left(1-\left|\eta_{1}\right|^{2}\right)^{\frac{1}{2}}\right\}} \frac{F^{z}(r, \eta, y)}{\left|\eta_{1}\right|^{\frac{n z}{m}}} d r d \eta_{1} d \eta^{\prime} .
\end{gathered}
$$

Here we set $\eta=\left(\eta_{1}, \eta^{\prime}\right)$, and we let $d \eta^{\prime}$ be the measure on the $(l-2)$-dimensional sphere $\left\{\left|\eta^{\prime}\right|=\left(1-\left|\eta_{1}\right|^{2}\right)^{\frac{1}{2}}\right\}$. If we make a change of variables in the above integral letting $\eta^{\prime} \rightarrow$ $\left(1-\left|\eta_{1}\right|^{2}\right)^{\frac{1}{2}} \eta^{\prime}$, we get

$$
\begin{aligned}
\widehat{B_{z, M}}(y)= & \psi(z) \int_{0}^{+\infty} r^{-\frac{n}{m} z+l-1} \chi\left(\frac{r}{M}\right) \int_{-1}^{1} \frac{\left(1-\left|\eta_{1}\right|^{2}\right)^{\frac{l-2}{2}}}{\left|\eta_{1}\right|^{\frac{n z}{m}}} \times \\
& \int_{S^{l-2}} F^{z}\left(r, \eta_{1}, \eta^{\prime}\left(1-\left|\eta_{1}\right|^{2}\right)^{\frac{l}{2}}, y\right) d \eta_{1} d \eta^{\prime} .
\end{aligned}
$$

We recall that the distribution $\frac{r^{-\frac{n}{m} z+l-1}}{\Gamma\left(l-\frac{n z}{m}\right)}$ is an entire function of $z$ which coincides with the Dirac distribution $\delta_{0}$ when $z=\frac{m l}{n}$, and that the distribution $\frac{\left|\eta_{1}\right|^{\frac{-n z}{m}}}{\Gamma\left(-\frac{z n}{2 m}+\frac{1}{2}\right)}$ is an entire function of $z$ which coincides with the $(k-1)$ th derivative of the Dirac distribution $\delta_{0}$ when $l=2 k+1$ and $z=\frac{m l}{n}$. With that in mind we consider a smooth function $\rho(t)$ which is $\equiv 1$ when $t \in\left(0, \frac{1}{4}\right)$ and is $\equiv 0$ when $t \in\left(\frac{3}{4}, 1\right)$, and we write

$$
\widehat{B_{z, M}}(y)=\psi(z)\left(\int_{-1}^{1} \rho\left(\eta_{1}^{2}\right) \cdots+\int_{-1}^{1}\left(1-\rho\left(\eta_{1}^{2}\right)\right) \cdots\right)=\psi(z) I_{1}(z, M, y)+\psi(z) I_{2}(z, M, y) .
$$

Since

$$
\left(1-\rho\left(\eta_{1}^{2}\right)\right)\left(1-\eta_{1}^{2}\right)^{\frac{l-2}{2}} \int_{S^{l-2}} F^{z}\left(r, \eta_{1}, \eta^{\prime}\left(1-\left|\eta_{1}\right|^{2}\right)^{\frac{l}{2}}, y\right) d \eta^{\prime} d \eta_{1}=F_{2}\left(r, \eta_{1}\right)
$$


is bounded and continous with respect to $r$ and $\eta_{1}$, we can write

$$
\psi(z) I_{2}(z, M, y)=\left.\Gamma^{-1}\left(-\frac{z n}{2 m}+\frac{1}{2}\right) \chi\left(\frac{r}{M}\right) \int_{-1}^{1} F_{2}\left(r, \eta_{1}\right) d \eta_{1}\right|_{r=0}
$$

Thus $\left|\psi(z) I_{2}(z, M, y)\right|$ is bounded by a constant with does not depend on $M$ and has at most exponential growth with respect to $\operatorname{Im}(z)$.

We shall now estimate $\psi(z) I_{1}(z, M, y)$. By our previous observations, the function

$$
\rho\left(\eta_{1}^{2}\right)\left(1-\eta_{1}^{2}\right)^{\frac{l-2}{2}} \int_{S^{l-2}} F^{z}\left(r, \eta_{1}, \eta^{\prime}\left(1-\left|\eta_{1}\right|^{2}\right)^{\frac{l}{2}}, y\right) d \eta^{\prime} d \eta_{1}=F_{1}\left(r, \eta_{1}\right)
$$

can be differentiated $l-1$ times with respect to $\eta_{1}$ and its derivatives are continous functions of $r$. Then, if $l=2 k+1$,

$$
\psi(z) I_{1}(z, M, y)=\left.\frac{d^{k-1}}{d \eta_{1}}\left(\chi\left(\frac{r}{M}\right) F_{1}\left(r, \eta_{1}\right)\right)\right|_{\eta_{1}=r=0}
$$

If $l \neq 2 k+1$, and if $h=\left[\frac{\operatorname{Re}(l)}{2}\right]$ we use the formula (3) in [GS], pg. 51, obtaining:

$$
\begin{gathered}
I_{1}(z, M, y)=\chi\left(\frac{r}{M}\right) \int_{0}^{+\infty} \eta_{1}^{-\frac{n z}{m}}\left(F_{1}\left(r, \eta_{1}\right)+F_{1}\left(r,-\eta_{1}\right)-\right. \\
\left.2\left(F_{1}(r, 0)+\frac{\eta_{1}^{2}}{2 !} \frac{\partial^{2}}{\partial \eta_{1}} F_{1}(r, 0)+\cdots+\frac{\eta_{1}^{2 h}}{(2 h) !} \frac{\partial^{2 h}}{\partial \eta_{1}} F_{1}(r, 0)\right)\right)\left.d \eta_{1}\right|_{r=0}
\end{gathered}
$$

Thus, $\left|\psi(z) I_{1}(z, M, y)\right|$ is bounded by a constant which does not depend on $M$. This shows that $\widehat{K_{z, M}}$ is bounded by a uniform constant. An easy adaptation of the above argument shows that $\widehat{K_{z, M}}$ converges to $\widehat{K_{z}}$ in distribution sense as $M \rightarrow \infty$.

This completes the proof of the theorem.

Acknowledgements : The authors wish to thank Eric Sawyer for financial support provided through his NSERC grant. The second author wishes to thank Professor Sawyer for numerous helpful conversations over the last two years, which had a profound influence on his work.

The authors wish to thank Fulvio Ricci for suggesting Theorem 3.1, and for related helpful suggestions. 


\section{References}

[MC] M. Christ, Restriction of the fourier transform to submanifolds of low codimension (Ph.D Thesis) U. of Chicago.

[DCI] L. De Carli and A. Iosevich, A restriction theorem for flat manifolds of codimension two, Illinois M.J. (to appear) (1995).

[Gr] A. Greenleaf, Principal curvature and harmonic analysis, Indiana Math J. 30 (1982), 519-537.

[GS] I. M. Gel'fand and G. E. Shilov, Generalized functions vol. 1, Academic Press. (1964).

[H] H. Hironaka, Resolution of singularities of an algebraic variety over a field of characteristic 0, Annals of Math. 79 (1964), 109-203, 205-326.

[I1] A. Iosevich, Maximal operators associated to families of flat curves in the plane, Duke Math J. Vol.76 (1994).

[I2] A. Iosevich, Maximal averages over homogeneous hypersurfaces in $\mathbb{R}^{3}$, Forum Mathematicum (to appear) (1995).

[IS] A. Iosevich and E. Sawyer, Oscillatory integrals and maximal averaging operators associated to homogeneous hypersurfaces, Duke M.J. (to appear) (1995).

[L] W. Littman, Fourier transforms of surface carried measures and differentiability of surface averages, Bull. Amer. Math. Soc. 69 (1963), 766-770.

[WR] W. Rudin, Functional Analysis, McGraw-Hill Inc. (1973).

$[\mathrm{P}]$

[TS] T. Shintani, On zeta function associated with the vector space of quadratic forms, J. of F.Sc. U. of Tokyo (1975), 25-65.

[So] C. D. Sogge, Fourier Integrals in Classical Analysis, Oxford University Press (1991).

[St] E. M. Stein, Harmonic Analysis, Princeton University Press 43 (1993). 